\title{
The Ethics of Care and the Newfoundland Paid Family Caregiver Program: An Assessment
}

by

\section{Margaret FitzGerald}

A thesis submitted to the Faculty of Graduate and Postdoctoral Affairs in partial fulfillment of the requirements for the degree of

Master of Arts

in

Political Economy

Carleton University

Ottawa, Ontario

(C) 2015, Margaret FitzGerald 


\begin{abstract}
The ethics of care has gained traction as a feminist normative lens from which to examine policies and policy issues. This thesis aims to contribute to this growing literature by employing a critical ethics of care lens to assess a new long-term care initiative in the province of Newfoundland and Labrador, Canada. This initiative, called the Newfoundland Paid Family Caregiver Program, allows eligible participants to pay family members for some care services. This analysis uncovers numerous tensions, both practical and theoretical, related to the way this program (re)organizes care. Specifically, the ways in which this program downloads caring responsibilities onto the family and monetizes caring relations is discussed. Finally, this analysis explores what care policies starting from the premises of a critical ethics of care might look like, and argues that such policies would have greater reach and positive consequences in terms of (re)producing healthy, flourishing lives for us all.
\end{abstract}




\section{Acknowledgements}

There are many people who have contributed to this thesis along the way; I am sincerely grateful to everyone who provided comments, critiques, and support during this process. In particular, I would like to thank Dr. Lisa Mills and my external reader, Dr. Christine Koggel, for providing thoughtful feedback; your contributions improved this work immensely. I would also like to thank the Social Sciences and Humanities Research Council, the Ontario Graduate Scholarship, and Carleton University for financial support during the writing of this thesis. Lastly, I wish to thank three people, without whom, this thesis would not exist.

To Dr. Fiona Robinson, thank you for your guidance and encouragement during this endeavor. Your work inspires me and I am grateful to have had this opportunity to learn from you.

To Ryan Murphy, my partner in every way, thank you for always supporting me. And, to Donna Coghill, for everything else. You provided the care I needed to complete this work. Thank you. 


\section{Table of Contents}

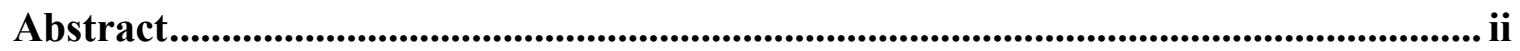

Acknowledgements ............................................................................................................................. iii

Table of Contents ............................................................................................................. iv

List of Tables ............................................................................................................................... vi

1 Chapter: Introduction ........................................................................................................ 1

2 Chapter: The Ethics of Care: A Historical Overview .................................................. 8

2.1 Chodorow, Gilligan and Psychological Theory......................................................... 9

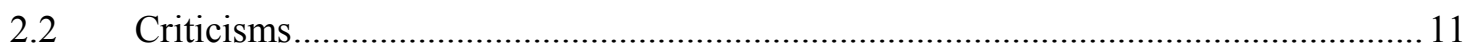

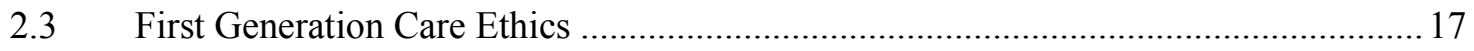

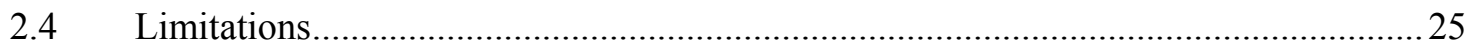

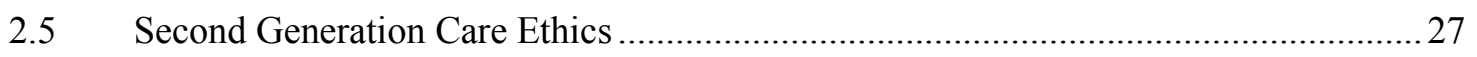

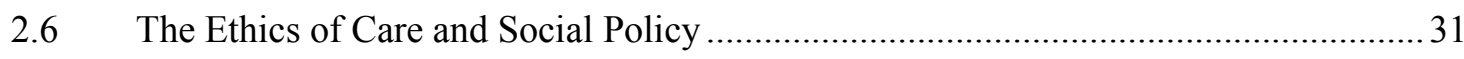

3 Chapter: A Critical Ethics of Care............................................................................ 35

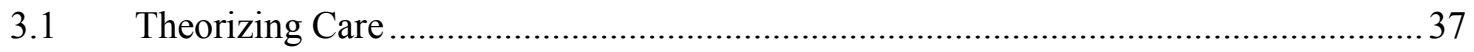

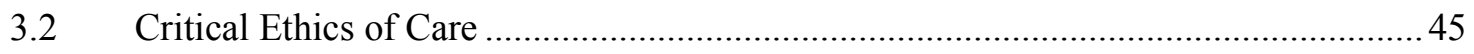

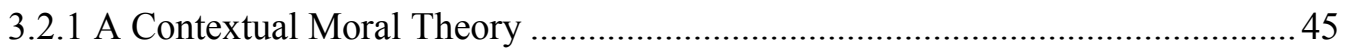

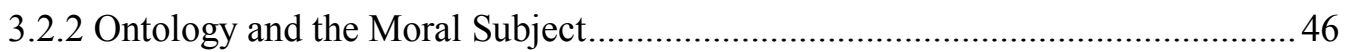

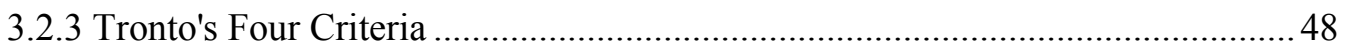

3.3 Methodological Approach: The Ethics of Care and Feminist Political Economy ........ 58

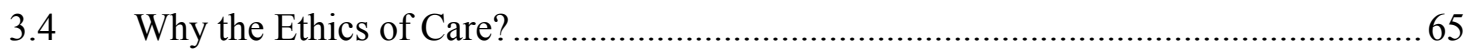

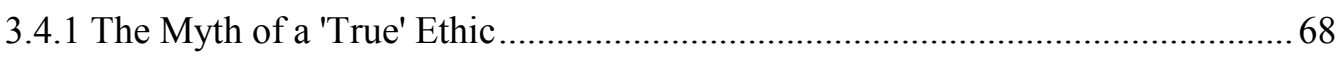

3.4.2 The Dangers of Equality, Individuality, and the Notion of Rights..................... 73 


\section{Chapter: Assessment of the Newfoundland Paid Family Caregiver Program from an Ethics of Care Perspective ............................................................................ 79}

4.1 The Case Study: The Newfoundland Paid Family Caregiver Program ........................ 80

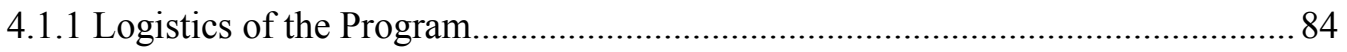

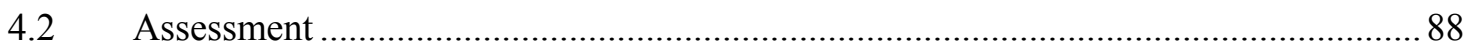

4.2.1 Contextual Sensitivity and the Moral Subject .................................................. 88

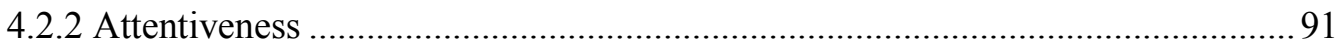

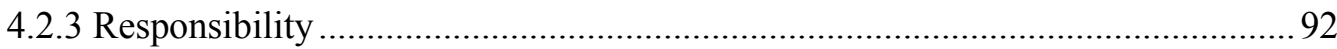

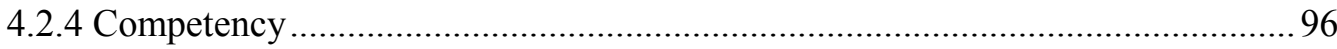

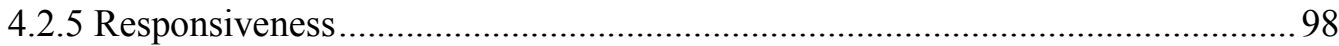

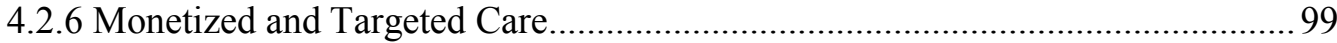

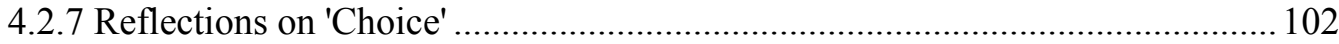

4.2.8 Care as a Social and Collective Responsibility ............................................ 105

4.3 A Final Thought: Imagining Alternative Policies................................................... 107

5 Chapter: Conclusion ..................................................................................................... 110

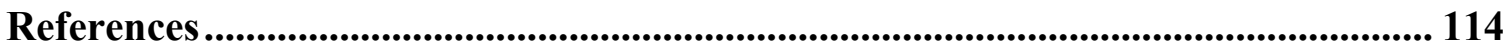




\section{List of Tables}

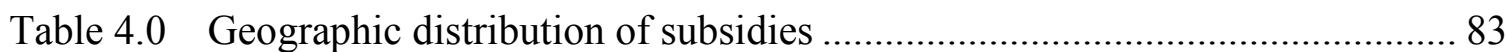

Table 4.1 Summary of maximum hours of care supported by the Newfoundland Paid

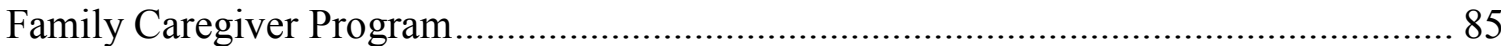

Table 4.2 Breakdown of household management/homemaking .................................. 86

Table 4.3 Detailed breakdown of maximum hours of care supported by the Newfoundland Paid Family Caregiver Program 


\section{Chapter: Introduction}

The province of Newfoundland and Labrador is facing significant challenges in terms of the provision of long-term care for the elderly population. Critical gaps in longterm care services, particularly in rural areas, have resulted from a combination of factors, including: shifts in demographic patterns, out-province migration, the decay of the traditional male breadwinner model, and ongoing cuts in social services. In response to this problem, the provincial ${ }^{1}$ Conservative Government is in the process of initiating a program whereby eligible home support clients in the province will have the option of paying family members for some personal care and behavioral support services. This program is called the Newfoundland Paid Family Caregiver Program. The pilot project for this program commenced on March 24, 2014. Although this program targets both senior citizens and adults with disabilities, this thesis will focus on the senior citizen population only. The vast literature on disability and care often addresses issues that do not frequently arise in elder care (for instance, disability literature highlights the oppressive history of care; cf. Kröger 2009; Williams 2001), and thus I have chosen to narrow my focus in this way for pragmatic reasons.

This thesis examines the Newfoundland Paid Family Caregiver Program using the ethics of care. Specifically, I address the following central research question: How does the Newfoundland Paid Family Caregiver Program reshape the realities of caregiving, care receiving, and care work, and what are the practical and theoretical implications of

\footnotetext{
${ }^{1}$ Throughout the entirety of this thesis, when I refer to any government or political party, I am referring to the provincial level of governance in Newfoundland and Labrador, unless stated otherwise.
} 
this? I use a critical ethics of care $^{2}$ as the methodological framework from which I

explore this question. I use the term 'critical' ${ }^{3}$ here to emphasize that the ethics of care, as

I formulate and employ it in this thesis,

emphasizes the importance of locating care within the context of the wider institutions and structures which shape the global order; understood in this way, [care ethics] can assist us in exposing the often hidden values and norms which reinforce and reproduce established exclusionary social practices and attitudes. [A critical ethics of care] attempts to show that, when taken as part of a larger, critical-relational approach to moral exclusion, care transcends its perceived limitations as an ethics which is relevant only in the context of physically and emotionally close personal relationships. (Robinson 1999, 110-111)

A critical ethics of care lens "brings into sharp relief the irreconcilability of neoliberalism's conception of the independent citizen with the real needs and desires of individuals as they pass through the life cycle" (Adkin and Abu-Laban 2008, 69); it understands care as a fundamental reality of all people's lives, in contrast to neoliberal ideology which takes autonomous individuals, abstracted from contexts and relations, as its ontological basis. I follow other feminist care ethicists (Hankivsky 2004; Mahon and Robinson 2011; Robinson 1999, 2011b; Williams 2001, 2010) in that I view critical ethics of care as a lens from which to understand politics and power dynamics. As Robinson argues, care and care relations are shaped by relations of power that vary through time, space and context. In this way, care relations not only reflect moral values, but are also of great political significance $(2011 \mathrm{~b}, 4-5)$. A critical ethics of care

\footnotetext{
${ }^{2}$ I use the term the ethics of care, as opposed to the ethic of care, because I believe that the plural term emphasizes that the ethics of care, as demonstrated in later chapters, is not defined and determined by strict, singular rules. Rather, the ethics of care provides a fluid framework from which to understand and address moral dilemmas. In my opinion, the plural term 'ethics' better captures this fluidity than the singular term 'ethic.'

${ }^{3}$ Not all care ethicists use the term 'critical.' Thus, throughout this thesis, I use the terms employed by particular authors when discussing their respective works.
} 
framework emphasizes the nexus of morality and politics, while also locating caregiving, care receiving, and relations of care as fundamental to the human condition. As opposed to relying on abstractions, critical ethics of care privileges the lived reality of all people, and seeks to illuminate and critically interrogate the complex ways social policies and care relations are intertwined. As the Newfoundland Paid Family Caregiver Program plays a pivotal role in shaping the care relations of the participants in the program, the use of an ethical and methodological framework which not only acknowledges these relations, but actually locates them as a primary reality for all people, allows me to understand how political decisions and policy formation affect the everyday lives of caregivers and care receivers. By employing this framework, this thesis contributes to the development of a comprehensive view of the effects of this program on long-term elder caregiving and care receiving in Newfoundland and Labrador by exploring how this program allows participants to address the needs, desires, and tensions that arise in caregiving and care receiving.

My analysis of the Newfoundland Paid Family Caregiver Program using a critical ethics of care lens reveals several salient points about this program. It highlights the fact that this program conceptualizes humans as independent, rather than relational beings: as such, I argue that under this program, moments of dependency, and indeed, ongoing dependencies, are framed as individual failings, rather than acknowledged as a fundamental reality of human life. My analysis also uncovers how this program is (re)shaping caregiving, care receiving, and care work by downloading the responsibility for care onto the family and (back) into the private sphere, in what perhaps could be called the 'refamilialization' of care, as opposed to the 'defamilialization' of care 
(Esping-Anderson 1999). This, I believe, not only reflects that care is not conceptualized as a collective, social responsibility under this program, but further indicates that this program works to perpetuate the characterization of care as a private concern. This normative assessment of the Newfoundland Paid Family Caregiver Program using care ethics further illuminates the nuanced ways in which this program is monetizing, as opposed to commodifying, care and care work. While both monetization and commodification involve the payment of wages to care workers, under monetization, access to care services is still publicly financed/provided, whereas under commodification, care services are accessed through the market. ${ }^{4}$ Additionally, this analysis shows how this program fails to consider the wider contexts, including systems of oppression, domination, and exploitation, in which all relations of care reside. This is problematic, for we do not have a full account of people's caring needs if we abstract them from their relationships and circumstances. Finally, because of these issues, I conclude that this program may not be facilitating quality, competent, consistent care.

My goal in this work is twofold. On the one hand, I seek to examine the Newfoundland Paid Family Caregiver Program so as to understand how this macro-scale policy program may affect the micro-care relations of the participants in the program. To this end, the core of this thesis is presented as a case study and analysis of the Newfoundland Paid Family Caregiver Program. On a larger scale, however, I hope to contribute to research and dialogue on care ethics in general. The ethics of care has gained much traction as a feminist normative perspective and methodology from which to

\footnotetext{
${ }^{4}$ I explain the difference between monetization and commodification in greater detail in chapter four.
} 
analyze, critique, and assess an array of issues, including women's work issues (Robinson 2006a), globalization (Hankivsky 2006), global justice (Robinson 2013b), military humanitarian intervention (Kyle 2013), human security (Robinson 2011b), gerontology and intergenerational care issues (Bozalek and Hooyman 2012), early childhood care and education (Adkin and Abu-Laban 2008), the construction of women's health in global health policy (Robinson 2013a) and social policy issues in general (Hankivsky 2004; Mahon and Robinson 2011; Sevenhuijsen 2003; Sevenhuijsen et al. 2006). This thesis aims to build on this growing literature by demonstrating how the ethics of care can usefully be applied to social policy issues, specifically those related to long-term elder care, thereby providing further evidence that this ethic can aid in developing a more robust understanding of the often far-reaching consequences that political decisions and processes have on our ability to maintain a flourishing world around us.

This thesis is organized as follows. In chapter two, I present a brief overview of the genealogy of the ethics of care, tracing its roots back to psychological theory, with a specific focus on the work of Chodorow (1978) and Gilligan (1982). I review some of the key contributions by a selection of authors from the 'first generation of care ethicists' and 'second generation of care ethicists.' I then present some of the more recent work on care ethics, in which scholars use the ethics of care to evaluate social policies. Importantly, while this chapter is largely meant to illustrate the evolution of the ethics of care, I also address some of the most prominent critiques of these works. In my exploration of these debates, I hope to contribute to a more nuanced understanding of the arguments for and against care ethics. I aim to contextualize my own use of the ethics of 
care by providing a historical account of the evolution of this framework over time, and by outlining the limitations of earlier care ethics theory. Lastly, I seek to demonstrate how such limitations have been overcome as scholars developed and expanded the framework of the ethics of care.

Building upon the historical literature review in chapter two, I present my own understanding of a critical ethics of care in chapter three. I provide definitions of some of the central terms in care ethics; in particular, I discuss a theory of care. I review the ontological foundation of the ethics of care, and spend time considering the moral subject of the ethics of care. I draw upon the works of care ethicists who have demonstrated the ways in which care ethics can be used to uncover critically aspects of social policies (i.e. Hankivsky 2004; Mahon and Robinson 2011; Robinson 1999, 2011b; Williams 2011). I also rely particularly upon the work of Joan Tronto (1993). Tronto's ethics of care involves four criteria: attentiveness, responsibility, competence, and responsiveness (1993). I argue that these criteria, combined with a critical feminist political economy methodology, can be used to evaluate various social policies, including the policy central to this thesis, the Newfoundland Paid Family Caregiver Program. In order to support this claim, I review each of these criteria in turn, so as to provide a detailed explication of these criteria and demonstrate how they can together facilitate an alternative reading of social policies in contrast to other policy analysis methodologies. I also show how a feminist political economy methodology can be combined with the ethics of care so as to facilitate such alternative readings.

Before moving into my analysis of the Newfoundland Paid Family Caregiver Program, chapter three also explains why normative analyses of social policies based on 
ethics are important, and I explain why I have chosen to use the ethics of care to evaluate the Newfoundland Paid Family Caregiver, rather than rely on mainstream ethical frameworks like liberalism, contractarianism, and communitarianism.

In chapter four, I evaluate the Newfoundland Paid Family Caregiver program using critical ethics of care, with specific reference to the criteria discussed in chapter three. This chapter first presents a background and overview of this program, and explains the logic and practicalities of this policy. I then evaluate the program using a critical ethics of care lens. I use several of the fundamental concepts of the ethics of care as points of entry to probe the logic and make up of this program. These concepts include contextual sensitivity and an examination of the moral subject represented in the program, and the four criteria of attentiveness, responsibility, competency, and responsiveness. By examining the program using each of these criteria, numerous tensions are revealed about this program and how it is (re)organizing care in the province of Newfoundland and Labrador. In particular, it becomes evident that this program is 'refamilializing' care while also monetizing care relations and care work.

Finally, in chapter five, I provide concluding remarks on this evaluation of the Newfoundland Paid Family Caregiver Program. I reassert that the tensions highlighted in chapter four support my thesis that the Newfoundland Paid Family Caregiver Program is 'refamilializing' care as well as monetizing care relations. I identify important directions for future research, and reiterate my argument that the ethics of care is a fruitful tool which can critically interrogate social policies and facilitate an alternative understanding of how political decision making and policy formation manifest in the relations of care which shape all our lives. 


\section{Chapter: The Ethics of Care: A Historical Overview}

The literature on the ethics of care can perhaps most accurately be traced back to psychological theory, specifically the work of Nancy Chodorow and Carol Gilligan (Fine 2007). Since then, many others have taken up the task to develop the ethics of care; such scholars have often been grouped into two categories: first generation care ethicists and second generation care ethicists (Hankivsky 2004; Mahon and Robinson 2011). In this chapter, I also identify an emerging literature that applies care ethics to social policy what might be called a 'third generation' of care ethicists. As the body of work on the ethics of care has evolved through these 'generations,' the concept has been expanded, debated, and perhaps most importantly, its potential to inform policy and thereby transform the lives of both caregivers and care receivers has been explored. In this first chapter, I review a selection of the key texts and articles that form this body of work to delineate both the development of the ethics of care, and to contextualize my own use of care ethics, which I describe in detail in chapter three.

This review is not meant to be exhaustive; however, the texts I have selected to present here are significant in that they highlight some of the central debates in the scholarship related to the ethics of care. By presenting these debates, I hope to provide an historical overview of both the strengths and weaknesses of this ethical framework. In addition, I seek to address some of the critiques of the ethics of care that have arisen over the years. In particular, I discuss the charge of 'essentialism,' which claims that the ethics of care naturalizes women as caregivers. I argue against such an understanding of the ethics of care; instead, I contend that the ethics of care is fundamentally about the 
acknowledgement that relations, specifically relations of care, are a primary reality of all our lives. The ethics of care emphasizes that these relations are shaped by social, cultural, political, and economic forces and power dynamics, and that arrangements of care affect real people in real, life-determining ways; by locating relationships in this way, the ethics of care allows for an alternative understanding of moral decisions, reasoning, and practice.

\subsection{Chodorow, Gilligan and Psychological Theory}

Chodorow's contribution to the development of the ethics of care arises from her work in psychological development theory, in which she examines how males and females experience relationships. Specifically, Chodorow's work challenges Freud's preOedipal theory by arguing that the "crucial differentiating experience in male and female development arises out of the fact that women, universally, are largely responsible for early child care and for (at least) later female socialization" (Chodorow 1974, 43).

Chodorow develops this argument by proposing that social and familial experiences differ between boys and girls because of historically and socially constructed familial divisions of labour: while young boys develop 'ego strength' through differentiation (the identification of boundaries) with their mother, Chodorow argues that girls also develop 'ego strength,' despite the fact that boundaries between mothers and daughters are not as substantial as those between mothers and sons $(1974,62)$. That is to say, whereas boys develop their ego by individualizing, and through processes of separation, Chodorow suggests that girls can (and often do) develop a strong ego through the opposite mechanisms: the continuation and strengthening of relational ties with their mothers. Notably, Chodorow argues that "women's mothering does not exist in isolation" 
$(1978,32)$, and so these relations are intricately informed by the sexual division of labour, including systems of male dominance, the varied forms of particular family systems, and the prevalence of the male breadwinner model $(1978,21)$. In this way, Chodorow's work demonstrates that relationships are both experienced and perceived differently by men and women and directly links these different experiences to "the institutional and normative social arrangements which perpetuate the hierarchical, structural differentiation of domestic and public spheres" (Robinson 1999, 14). Chodorow sees differences in relations as stemming from the societal construction in which the family division of labour allocates mothering to women, thereby defining the meaning of woman or womanhood itself. Chodorow thus not only provides a theory which explains developmental differences between boys and girls, but writes against the masculine bias of psychoanalytical theory, in which women are theorized as having weaker ego boundaries than men (Gilligan 1982, 8).

Building on Chodorow's work, Gilligan's scholarship explores the differences between the moral development of men and women. Challenging Kohlberg's six stages of the development of moral judgment (1981), she uses interviews with both men and women, of a variety of ages and through time, to illustrate that "women not only define themselves in a context of human relationship but also judge themselves in terms of their ability to care" $(1982,17)$. To demonstrate using a well-known example, Gilligan interviewed two school-aged children, Amy and Jake, and posed a moral dilemma: should a man steal a life-saving medication for his dying wife?

Interestingly, Jake was firm in his answer that the man should steal the medication, as 'lives are worth more than money,' and he was assured that the law would 
understand (1982, 26-28). Amy, in contrast, was much less decisive, and far more concerned with how each action could affect the relationship between the man and his wife:

If he stole the drug, he might save his wife then, but if he did, he might have to go to jail, and then his wife might get sicker again, and he couldn't get more of the drug, and it might not be good. So, they should really just talk it out and find some other way to make the money. $(1982,28)$

As Gilligan notes, Amy sees "in the dilemma not a math problem with humans but a narrative of relationships that extends over time" $(1982,28)$. Based on this, and other observations like this, Gilligan asserts:

Women's construction of the moral problem as a problem of care and responsibility in relationships rather than as one of rights and rules ties the development of their moral thinking to changes in their understanding of responsibility and relationships, just as the conception of morality as justice ties development to the logic of equality and reciprocity. Thus the logic underlying an ethic of care is a psychological logic of relationships, which contrasts with the formal logic of fairness that informs the justice approach. $(1982,73)$

It is here that the ethics of care first explicitly appears in the literature as a description of an ethical framework which emerges from a relational understanding of the world and moral dilemmas.

\subsection{Criticisms}

These works were not received without criticism, and continue to be controversial today. Chodorow's work has been critiqued for its heterosexual bias (Rich 1980), its lack of discussion and consideration for race, class, and other structural factors (Coser 1981; Gewertz 1984; Lorber 1981; Spelman 1988), evidentiary and methodological concerns (Rossi 1981), and for her reliance on objects-relation theory (Bart 1981; also see Robinson 1999, 14-15). Gilligan has been accused of exaggerating the differences 
between the moral development of women and men (Broughton 1983; Senchuk 1990), of methodological issues (Luria 1986), and of being 'essentialist,' by naturalizing women's supposed affinity for relations and care (Kerber 1986). Furthermore, many feminist scholars take issue with Gilligan's work for its lack of engagement with other perspectives and positionalities, as influenced by identity markers like class, race, and sexual orientation (cf. Fraser and Nicholson 1989; Stack 1986). While both Chodorow (1981) and Gilligan (1986) have replied to their critiques, it is these last two issues which particularly concern me: (1) the accusation that Chodorow and Gilligan's work naturalizes women as caring and (2) that this work essentializes all women as having the same affinities to care, regardless of different contexts and positionalities.

In much of the literature, these criticisms have been lumped together under the accusation of 'essentialist.' As Heyes, citing Martin (1994), writes:

$[\ldots]$ any feminist theory that is variously determinist, exclusionary, ahistorical; that fails to recognize diversity among women; that falsely generalizes, reifies femininity, or commits any other related methodological sin tends to be dubbed "essentialist." $(1997,144)$

I contend that such an all-encompassing term is not useful here. Rather, I suggest that the majority of the critiques that fall under the umbrella of the 'essentialist' complaint can be divided into two categories. The first is the claim that women are natural caregivers; the second is the critique that Chodorow and Gilligan's work does not effectively grapple with differences that exist due to the particular and unique situations and positionalities that women occupy; in other words, that these works are western-centric. ${ }^{5}$ I contend that

\footnotetext{
${ }^{5}$ In fact, some scholars actually describe these works in this way, rather than referring to them as 'essentialist.' For instance, Gewertz (1984) criticizes Chodorow for failing 'to consider adequately nonWestern views of the self' (615).
} 
the first of these claims is unjustified, while the second of these is certainly a central problem in these works.

As Tronto notes, “the equation of Gilligan's work with women's morality is a cultural phenomenon, and not of Gilligan's making" $(1987,64)$, and I would argue that the same comment can be extended to Chodorow's work as well. A close reading of both of these works illuminates that neither of these authors suggest that women are naturally or biologically better caregivers (or even naturally caring at all). Throughout her work, Chodorow refers to the structural ways in which women are situated to care (for instance, 1978, 21), and Gilligan notes:

The different voice I describe is characterized not by gender but theme. Its association with women is an empirical observation, and it is primarily through women's voices that I trace its development. But this association is not absolute, and the contrasts between male and female voices are presented here to highlight a distinction between two modes of thought and to focus a problem of interpretation rather than to represent a generalization about either sex... No claims are made about the origins of the differences described or their distribution in a wider population, across cultures, or through time. Clearly, these differences arise in a social context where factors of social status and power combine with reproductive biology to shape the experience of males and females and the relations between the sexes. $(1982,2)$

Gilligan does not resort to a biological explanation of the 'caring voice' she identified in her research; she simply acknowledges this voice. The question of how this voice came to be is, as she admits, beyond the scope of this particular work. Nonetheless, while Gilligan does not examine the source of this difference, she does indicate that she personally believes that social contexts and power relations are important factors that contribute to this different voice. I find it unfounded to conclude from this that Gilligan regards women as natural caregivers. 
Even more telling, in my opinion, is the very use of the term 'a different voice.' Like the title of the book, and as Gilligan makes clear elsewhere $(1986,327)$, the central message behind this work is not the identification of a 'woman's voice,' but a 'different voice.' Gilligan's different voice is perhaps best described when she explains how this different voice conceptualizes the moral problem:

In this conception, the moral problem arises from conflicting responsibilities rather than from competing rights and requires for its resolution a mode of thinking that is contextual and narrative rather than formal and abstract. This conception of morality as concerned with the activity of care centers moral development around the understanding of responsibility and relationships, just as the conception of morality as fairness ties moral development to the understanding of rights and rules. $(1982,19)$

The 'different voice' which conceptualizes moral problems in such a way need not be 'female.' While Gilligan is often (mis)interpreted as suggesting "that it is a statistically provable fact that men and women have different moral voices" (Robinson 1999, 18), my reading of In a Different Voice suggests that Gilligan is critiquing theories of morality which draw upon abstract principles to conceptualize moral problems as opposed to the earthly relations which comprise all of our lives. In this way, my reading closely follows that of Hekman (1995). Hekman argues that there are two ways of reading Gilligan's work; the first reading suggests that women have an essentially different voice, and that this is somehow a 'truer voice' than those heard in other, male-biased theories, while the second reading challenges the construction of morality itself $(1995,5)$.

[...] Gilligan is well aware that theorists such as Kohlberg have listened to women's stories, but, because they employed the interpretive framework of separate selves, they were forced to classify these stories as deficient and those who told them as lacking the qualities necessary for moral agency. What Gilligan is proposing is an alternative framework in which these women's stories are interpreted as genuine moral statements. If, as Gilligan proposes, we interpret relationship, care, and connection as integral to human life and development, then 
we will interpret women's stories as genuinely moral narratives, distinct from, but every bit as moral as, those based on abstract principles. (emphasis in original, $1995,7)$

Following this second reading, Gilligan's work is clearly not concerned with the 'natural' differences, whether real or imagined, between men and women. Instead, Gilligan is critiquing the male-biased epistemology which legitimizes moralities based on autonomous individuals, and which privileges those who use such moralities and abstract principles to guide their reasoning and actions. While it may be that historically and materially, the people who promote such moralities have been male, this does not mean that men cannot draw upon moral conceptions which start from a relational ontology and epistemology. Indeed, there are many examples of cultures, groups, and individuals which espouse such ontology and epistemology. For instance, Wilson (2008) describes how Indigenous epistemology and ontology are both based upon relationality. Even the classic novella $A$ Christmas Carol, written by a (white, British) man and originally published in 1843, iterates a sense of morality based on relation. Scrooge has chosen to live his life as an autonomous and fully self-sufficient human, and while he certainly has not gone out of his way to help anyone, he also has not done direct harm to others. Yet, he is visited by three ghosts who warn him that he is doomed if he does not foster caring relations with those around him. As a moral tale which is often told to warn against greed, I suggest that the true moral message of this tale is the importance of acting in ways which nurture healthy and mutually beneficial relations. Clearly, such a relational morality need not be, nor necessarily is, a woman's morality.

The critique that these works are western-centric, on the other hand, is, in my opinion, a much more substantial concern, and I agree wholeheartedly that this is a 
shortcoming of both of these works. Chodorow's concept of mothering is undoubtedly a white, heterosexual, middleclass concept of mothering, and Gilligan's interviews are also only with white American women. ${ }^{6}$ Because of this, aspects of both Chodorow's and Gilligan's work are problematic. ${ }^{7}$ Gender cannot be separated from class, sexuality, race, etc., and if we treat one group of women's experience or ideology as definitive, we risk erasing women's diversity and miss the opportunity to understand the unique realities of particular women's lives. Yet, while I agree that "in no way should the racist, classist, or heteronormative bases of some of this theory be minimized" (Heyes 1997, 144), I would also suggest that the fact that these works are western-centric does not void them of all value. Like all scholarship, the body of work on the ethics of care had to start somewhere. And while this does not excuse the western-centric nature of these pieces of research, I believe that the central insight, that moral decisions and moral practice can be framed and evaluated in terms of relations, is not limited to Western, middleclass, white, heterosexual worlds. Rather, a morality arising from the material reality that relations of care are fundamental for all humans can be a universally useful ethic which at the same time prioritizes and respects the particularities that arise in different times and contexts. The western-centrism of these works can be overcome by employing feminist methodologies, such as intersectionality (cf. Hankivsky 2014) and critical feminist political economy (cf.

\footnotetext{
${ }^{6}$ Gilligan has, in fact, expanded her work to try and address this shortcoming by conducting interviews with women who occupy different positionalities. For more information, please see her later work, particularly Between Voice and Silence: Women and Girls, Race and Relationships (1997), authored with Jill McLean Taylor and Amy Sullivan.

${ }^{7}$ In chapter four of Inessential Woman, Spelman (1988) provides a particularly insightful critique of Chodorow's work by arguing that gender formation, as Chodorow outlines, is not "neatly separable from other aspects of identity such as race and class," especially if, "as Chodorow insists, the acquisition of gender occurs in and helps perpetuate the 'hierarchical and differentiated social worlds' we inhabit" (1988, 82).
} 
Robinson 1999), which emphasize the unique experiences of individuals based upon their particular situations, and in relation to the power dynamics and systems of domination, oppression, and exploitation that influence their lives. ${ }^{8}$ Despite the western-centrism of these works, I contend that the most significant insight from these works is that they locate relations as legitimately comprising the moral foundations of certain reasoning and behaviors, and it is this insight which I believe has most shaped subsequent theories of care ethics, including those outlined below.

\subsection{First Generation Care Ethics}

The first generation of care ethics literature shares a marked departure from the psychoanalytical theory discussed previously, albeit while also branching off in different ways. Nel Noddings (1984) work on moral education, Sara Ruddick’s (1989) book on maternal morality, and Virginia Held's (1993) turn to culture and ethics are noted as some of the most significant texts of this generation (Mahon and Robinson 2011).

In Caring: A Feminine Approach to Ethics and Moral Education (1984), Noddings explores how the ethics of care may be applied in an educational context. Noddings actively formulates the ethics of care by exploring the relationship between the 'one-caring' (caregiver) and the 'cared-for' (care receiver). She argues that "apprehending the other's reality, feeling what he feels as nearly as possible, is the essential part of caring from the view of the one-caring" $(1984,16)$, and this ability to leave our "own a priori frame of reference" $(1984,24)$ is what causes us to act. If we understand the cared-for's needs and perspective, we will be motivated to respond: this,

\footnotetext{
${ }^{8}$ I elaborate on this extensively in chapter three.
} 
contends Noddings, is the difference between care ethics and an ethic of principle, in which principles, reason, and justified truths motivate us to act $(1984,45)$.

It is interesting to consider that many of the vignettes Noddings uses to illustrate her ethics of care are centered on the mother-child relationship (i.e. 1984, 43-44; 87-89; 130). This focus makes sense for Noddings because she describes care as involving an 'intangible something' $(1984,20)$, which she compares to the intangible affections which motivate artists like Mozart, Gauss, and Miró in their artistic endeavors $(1984,36)$. Such intangible feelings, for Noddings, create the capacity to care: "We do not begin by formulating or solving a problem but by sharing a feeling” $(1984,31)$. Such feelings, perhaps, are never more evident than within familial relations, which explains Noddings' focus on maternal relations. However, I suggest that it is this reliance on feelings which limits Noddings' work on care ethics, especially if care ethics is to be thought of as a political project.

Feelings, so very often, require immediate and close contact. However, formulating the ethics of care based on 'shared feelings' does more to relegate this ethic to the private sphere than to demonstrate how it can provide a meaningful ethical framework from which to address moral problems in the public sphere. And while Noddings argues that these feelings can be extended by viewing the world in chains, in which one's close relations are connected to others $(1984,49)$, and suggests that we should consider broader problems as if they were happening to a loved one $(1984,54)$, I counter that the fundamental logic behind such claims privileges private relations of care. By extension, this limits the potential for the ethics of care to be taken seriously in the political arena. As Sowerby argues, Noddings' ethics is unable to account for ethical 
relations with the non-intimate (1993), and "leaves starving people in a distant land outside the realm of moral consideration" (Hoagland 1990, 113). While Noddings description of care as "practical, made for this earth" $(1984,99)$, and her placement of care ethics in lived experience, practical human needs, and material reality (rather than the abstract logic of reason) is a strong contribution to the ethics of care, her focus on intangible feelings and personal relations limits the potential of this ethic to the personal or private domain by personalizing the ethics of care.

Ruddick's Maternal Thinking (1989) also formulates the ethics of care from a material framework. Asserting that all knowledge is constructed and dependent on the context and frame of reference in which it is (re)produced $(1989,128)$, Ruddick's ethics of care shares a foundation with Noddings' work, in that the social, political, and economic reality, and how individuals are positioned and understand that reality, is at the heart of this ethical framework for both of these scholars. However, Ruddick's work also differs from Noddings' work in significant ways, particularly in that it explicitly ${ }^{9}$ focuses on the maternal perspective or point of reference. While this is an interesting perspective, it has also been the source of most of Ruddick's criticisms.

In particular, while noting at the very beginning of her book that "throughout history and still today women assume disproportionately the responsibilities of caring for children" (1989, xi), Ruddick's focus on mothers as the sole actors in child care has, arguably, done more to essentialize and naturalize women as caregivers than to challenge

\footnotetext{
${ }^{9}$ That is, while I have pointed out that Noddings relies on the mother-child relation to demonstrate, and indeed even formulate, her ethics of care, she does not explicitly address this framing by acknowledging the maternal standpoint more generally. Ruddick is very clear that she is using the maternal standpoint as the point of departure for her ethics.
} 
this perception (Mahon and Robinson 2011, 4). Dietz argues that maternal thinking "distorts the meaning of politics and political action largely by reinforcing a onedimensional view of women as creatures of the family" $(1985,20)$. Furthermore, several scholars have pointed out that theories which rely on the idea of 'mother' are often ethnocentric and, like the western-centrism present in Chodorow and Gilligan's works, cannot be universalized to all women (Bailey 1994, 1995; Collins 1994; DiQuinzio 1993; for an overview see Keller 2010). Despite these points, I suggest that a close reading of Ruddick's work demonstrates that unlike Noddings, who does prioritize the familial relations of care that arise from what appears to be natural maternal love $(1989,31)$, Ruddick conscientiously works to avoid such a formulation of care ethics, and also takes steps toward acknowledging the diversity of motherhood as shaped by different cultural, social, political, and economic contexts.

Firstly, I contend that Ruddick's maternal standpoint does not relegate care ethics to the household because Ruddick is adamant that mothering does not belong to family/domestic realm alone:

Contrary to myth, mothers do not work in private. They are always in public, in doctors' offices and clinics, supermarkets and welfare offices, courthouses and schools, movie houses and amusement parks. $(1989,35)$

She sees maternal practice as something which happens within and across both the private and public sphere. Because of this, Ruddick's maternal thinking and ethics can transcend the imagined dichotomy between public and private. It challenges the notion that mothers are 'creatures of the household,' and highlights the fact that maternal care work is embedded in the wider social-political-economic context. The potential to subvert the private/public divide is further strengthened by Ruddick's adoption of feminist 
standpoint theory. This theory does not naturalize women (or mothers specifically) as caring, but rather,

fight[s] against the exploitation and abuse of women caretakers while valuing the particular knowledge that women acquire from their suffering of and resistance to oppression... [The] focus is not in the first place on gender but on the work itself and the political conditions in which it is undertaken. $(1989,133)$

By using standpoint theory, Ruddick sees maternal practice as work that "has been performed by women and has created 'women' as they are” (emphasis added, 1989, 133). She does not imply that maternal thinking is a natural part of 'being' a woman. Rather, Ruddick's maternal thinking highlights how maternal work, which through relations of power has often been relegated to women in the household, and which has often resulted in the devaluation and oppression of such women, can provide the basis of an alternative moral and epistemological vision $(1989,133)$ that is not divorced from the ways mothers often experience the world due to the social and political conditions in which mothering takes place.

Finally, the claim that Ruddick's maternal thinking cannot be universalized because Ruddick's maternal thinking is ethnocentric is valid in many regards. However, I assert that Ruddick attempts to account for this issue by bringing her own biases to the fore and practicing reflexivity (Finlay 2002). Ruddick adopts a semi-autoethnographic style in this book, and attempts to clearly articulate her own privileges and positionality (i.e. 1989, 3-12; 53-57). She prefaces many of her thoughts with phrases like "in most of the cultures I consider" $(1989,42)$, thereby acknowledging that such thoughts and observations cannot necessarily extend across all contexts. Furthermore, Ruddick 
recognizes that even when one identifies one's privilege(s), the experiences of one person can never be universal:

Although I try to correct for these biases, I cannot speak for any other mother [...] Many kinds of maternal stories need to be told: by heterosexual, gay, and lesbian mothers; by mothers who are coupled, single, or live in groups; by mothers separated from their children's female or male biological parent; by mothers who are celibate or monogamous or who have many sexual partners. $(1989,54)$

Ruddick's call 'for the telling of more maternal stories, from different cultural, racial and economic perspectives" (Rumsey 1990, 125) further suggests that Maternal Thinking is an attempt to develop an alternative morality based upon the unique material lives of mothers, and not based on a universal 'experience of motherhood.' Because of the fact that Ruddick acknowledges these differences, Ruddick's work cannot be read as an attempt to speak for "Everywoman" $(1989,65)$. This is not to suggest that Ruddick's work is without flaws; however, I believe that because Ruddick is clear that there is no such thing as one story of motherhood, the lack of engagement with other particular and unique maternal practices in Ruddick's theory can be overcome through the telling of a variety of stories about mothering. For this reason, I see Ruddick's work as far less problematic than that of Noddings', who is neither forthcoming with her biases nor actively calling for other stories of care.

Lastly, in Feminist Morality: Transforming Culture, Society, and Politics, Held investigates the role of culture and morality, and makes important contributions to the development of care ethics. Noting that morality implicitly involves

living our lives and actively shaping our relationships with others rather than accurately registering and theorizing about the impressions made upon us by what some take to be an external world $(1993,22)$, 
Held sees culture as a central component informing our ethics. In her definition of culture, Held includes "the material forms of the production and development of culture, and of the material lives of those who make culture and are shaped by it" $(1993,3)$; this indicates that Held, like Noddings and Ruddick, sees material reality as central to ethical inquiry. Held also begins to frame morality and ethics as having the potential to emancipate women. Seeing feminism as a way to shape culture, and thereby shape a new understanding of ethics based on care, Held contends that this may have the power to challenge the oppression of women:

Moral theories, $[\ldots]$, should give us guidance in confronting the problems of actual life in the highly imperfect societies in which we live. We need moral theories about what to do and what to accept here and now. Ideal theories of perfect justice or purely rational theories for ideal societies leave the problems of what to do here and now unsolved, even unaddressed. $(1993,23)$

Given that the ethics of care, as a moral theory, is shaped by material reality and lived experiences, Held sees this as the antidote to the 'ideal theories' that do not address 'the problems of actual life,' and thereby feels that it has potential to confront women's oppression.

Held also prioritizes human relations, which distinguishes her morality from traditional perspectives.

The individual assumptions of liberal theory and of most standard moral theory are suspect. Even if we were freed from the debilitating aspects of dominating male power to 'be ourselves' and to pursue our own interests, we would, as persons, still have ties to other persons, and we would, at least in part, be constituted by such ties. $(1993,58)$

Held recognizes the way human relationships colour the human experience and are central to all our lives, and throughout her work it is evident that she relies upon a relational ontology, like the care ethicists mentioned above. Importantly, while Held 
highlights the importance of relations, she also notes that "to argue for a view of the self as relational does not mean that women need to remain enmeshed in the ties by which we are constituted" $(1993,62)$. In other words, while the self is a relational being, it is clear that Held views relations of oppression, domination and exploitation as relations which must be broken and/or drastically reshaped. I also suggest that by acknowledging that not all relations are healthy, Held's relational ontology creates space to fully acknowledge both parties in the relationship. Unlike Noddings' relational ontology, which focuses on the 'one-caring,' Held's reminder that relations often embody oppressive institutions and systems emphasizes that even within a relational ontology, it is important that both of the individuals in a relation be heard. This is not to say that a relational ontology is reducible to the individual; rather, it is to acknowledge the fact that all relations involve a distribution of power. This distribution, often depending upon the way in which political structures, power dynamics, and social constructs have informed this relation, is not necessarily equal. Held reminds us that a critical ethics based upon a relational ontology must not equate/reduce all relations to an automatic status of 'good.' Instead, we must scrutinize all relations to understand whether or not they allow for the healthy flourishing of all of the individuals impacted by said relation.

Finally, Held's feminist moral theory challenges two binaries that are prevalent in traditional moral theories: the binary between reason and emotion $(1993,49)$, and the public/private divide $(1993,54)$. The divide between reason and emotion, whereby traditional moral theories suggest that abstract reasoning is the only way to make moral decisions, does not make sense from a materialist standpoint. Reasoning without emotion requires abstraction, a removal of the contextual specificity to follow a conceptual ideal. 
However, as Held argues, an ethics built on a relational ontology and from a material understanding of the world depends upon the very real relations and structures that uniquely inform our lives. Held's feminist ethics thereby destabilizes the constructed divide between reason and emotion, as moral action must be based upon the particularities of a situation, which often involve feelings of empathy and caring that influence and guide our actions $(1993,51)$. Similarly, Held's morality challenges the public/private dichotomy by again focusing on the material reality. The so called private sphere does not exist as "an island beyond politics" $(1993,54)$. Family life, in whatever form, is influenced greatly by societal structures and political processes. In return, the relations that are often thought of as belonging to the private sphere shape language and culture, form human social personhood, and develop morality $(1993,55)$. In this way, the material reality and political economic structures inform the 'private' life, while the 'private' life comes to inform the 'public' life. In Held's view, morality cannot be designated to the public or private sphere; rather, it destabilizes the very notion that such a distinction can be made at all.

\subsection{Limitations}

Hankivsky, in my opinion, summarizes the limitations of the work of the first generation of care ethicists' best when she argues that first generation care ethicists seem to mistake a feminist ethics of care for a feminine ethics of care. For instance, Noddings, Ruddick, and even Held (cf.1987) take the mother identity as the starting point for the ethics of care, and while the argument can be made that mothering need not be a feminine identity (Ruddick 1989, 40), it has been historically and socially constructed as such. As bell hooks writes: 
Because the world 'maternal' is associated with the behavior of women, men will not identify with it even though they may be behaving in ways that have traditionally been seen as 'feminine.' [...] Seeing men who do effective parenting as 'maternal' reinforces the stereotypical sexist notion that women are inherently better suited to parent, that men who parent in the same way as women are imitating the real thing rather than acting as a parent should act. $(2000,139$; also quoted in Ruddick 1989, 43)

An ethics of care which starts from the maternal standpoint (or any other 'feminine' identity for that matter) risks pigeonholing the ethics of care as a feminine morality. This 'feminine' morality can then be positioned in opposition to masculine moralities which rely on abstract principles, thereby (re)producing "the mode of arguing in binary oppositions with which Western thinking is so thoroughly permeated" (Sevenhuijsen 1998, 12). This severely limits the potential for the ethics of care to provide a meaningful ethic for all people.

This, however, is not to say that feminist issues are not an important aspect of the ethics of care. Following Sevenhuijsen, I believe that the ethics of care can, and should, be thought of as a feminist ethics of care: ${ }^{10}$

[...], although it is quite possible to conceptualize the ethics of care without attaching the adjective 'feminist' to it, I still prefer to add this word wherever possible. In the first place it shows the source of my inspiration and aims: further reflection on the feminist attitude to care and ethics, and the decoding of the gender-load inherent in established moral and political philosophy. Both care and ethics are indeed so interwoven with gender that we cannot do without continuous reflection on feminist interpretations and feminist points of view. But I also include the adjective 'feminist' for the sake of historical accuracy. Feminist thinkers, after all, have, since the 1980s, most clearly drawn attention to an ethics of care and responsibility. In doing this, they had a political objective: to expose the sexism and gender-blindness in moral philosophy and to give space to the moral considerations of women and 'feminine' moral voices and considerations. $(1998,34-5)$

\footnotetext{
${ }^{10}$ I elaborate on this in chapter three.
} 
By shifting the focus from feminine morality to feminist morality, second generation care ethicists honour the history of the ethics of care and remind us that the ethics of care can help achieve feminist aims. They also create space for the ethics of care to be applicable across gender identities; I now turn to a more detailed account of second generation care ethics, and how this body of work has shaped the ethics of care.

\subsection{Second Generation Care Ethics}

Joan Tronto's book Moral Boundaries: A Political Argument for an Ethic of Care (1993) distinguishes itself from first generation care ethics literature in three major ways. For one, I suggest that Tronto provides the first explicit and detailed definition of the ethics of care. Secondly, Tronto works to politicize care ethics by arguing that we must think of care ethics not just in terms of morality but also within a political context. Lastly, Tronto moves away from the previous literature which arguably conveys care ethics as essentially a women's morality, by demonstrating how inherent power structures situate women in the positions by which they have come to be associated with a care-based morality.

Tronto's definition of the ethics of care is based on the ethics of care as a practice, rather than as a list of abstract rules. It articulates that morality means that we strive to foster healthy caring relations and meet the needs of caring for oneself and others as they change throughout our life course. To facilitate this understanding of care ethics as a practice, Tronto presents four moral qualities which are also practice-based. ${ }^{11}$ The elements of the ethics of care, according to Tronto, include attentiveness, which

\footnotetext{
${ }^{11}$ These are described in detail in the next chapter.
} 
highlights the importance of recognizing needs (1993, 127); responsibility, which she argues must be a flexible understanding of responsibility, rather than the stricter connotations implied by 'obligation' $(1993,131,133)$; competence, which Tronto asserts "align[s] this approach with moral consequentialism" $(1993,133)$, in that competently providing/receiving care allows us to evaluate if the care relation is adequate $(1993,133)$; and finally, responsiveness, which implies that both caregivers and care receivers require safe spaces to respond to the care relation $(1993,134)$. Finally, Tronto notes that while these elements can be considered individually, the integrity of the ethics of care relies on the fact that these elements are intertwined and interacting in any care relationship.

Beyond simply defining the ethics of care, Tronto seeks to politicize care ethics because she argues that "if the study of moral development proceeds without any understanding of its political context, then it will necessarily reinforce the views that the powerful are morally privileged" (61). Tronto argues that focusing on the ethics of care as 'women's morality' is counterproductive in this sense, and reinforces three 'moral boundaries:' the boundary between morality and politics, the 'moral point of view boundary,' and the public/private boundary (1993, 6-11). Specifically, the moral-political boundary arises from the conception that morality and political concerns are two different spheres, and Tronto fears that framing the ethics of care as a 'women's morality' emphasizes that this ethical framework does not belong in the political sphere. The moral point of view boundary stems from the idea that moral judgments must be objective; women's morality will not be taken seriously here, Tronto argues, because "any account of morality that draws upon emotion, daily life, and political circumstance, will necessarily seem corrupted by non-rational and idiosyncratic incursions within this 
world" (1993, 10). Finally, the public and private life boundary is problematic if the ethics of care is framed as women's morality, as Tronto suggests that

[...] even if women could demonstrate that they possess a unique set of moral qualities and perspectives, these perspectives could easily be contained by arguing that they have no place in a realm of life that extends beyond the private sphere of friends and family. $(1993,10)$

As a result, Tronto suggests that the 'women's morality' terminology be exchanged for a language of the ethics of care, which she believes can more fully capture how women came to be excluded from public life in the first place, and how certain socially and politically constructed boundaries work to keep women excluded (1993). Only by moving away from the idea that the ethics of care is somehow specifically female does Tronto see a way to contextualize the ethics of care within politics and to understand how relations of care are already informed and structured by political inequalities.

Selma Sevenhuijsen's work (1998) is also concerned with politicizing care ethics. Sevenhuijsen argues that if the ethics of care is to acquire any notable political meaning without being type-cast as identity politics, locating the ethics of care within conceptualizations of citizenship is of the utmost importance. She writes:

When the ethics of care is located within such a notion of citizenship, discursive space is created for carers to bring their expertise and moral considerations into public debates without this being associated with a fixed caring identity or with associated claims to moral truth or moral goodness. $(1998,15)$

Like Tronto, Sevenhuijsen seeks to politicize the ethics of care and to move away from any 'fixed care identities' previously associated with this moral framework. Sevenhuijsen 
also demonstrates the differences between the ethics of care and traditional moral frameworks. $^{12}$

First of all, the ethics of care involves different moral concepts: responsibilities and relationships rather than rules and rights. Second, it is bound to concrete situations rather than being formal and abstract. And third, the ethics of care can be described as a moral activity, the 'activity of caring,' rather than as a set of principles which can simply be followed. The central question in the ethics of care - how to deal with dependency and responsibility - differs radically from that of rights ethics: what are the highest normative principles and rights in situations of moral conflict? $(1998,107)$

These distinctions are crucially important when one considers that Sevenhuijsen particularly 'paved the way' for the next generation of care ethics scholars, who focus on care ethics and social policy. The final two chapters of her book Citizenship and the Ethics of Care: Feminist Considerations on Justice, Morality and Politics (1998) are case studies (also see Sevenhuijsen et al. 2006). The first examines law and child custody from an ethics of care perspective, and in the second, she seeks to assess healthcare policies in the Netherlands using the ethics of care. These case studies provide a preliminary guide for how to approach policy evaluation from an ethics of care perspective. I believe that because Sevenhuijsen clearly distinguishes between the ethics of care and other moral frameworks, her application of care ethics to social policy issues is strengthened: she effectively highlights how the ethics of care starts from different premises and asks different questions than other moral theories, thereby demonstrating how the ethics of care can illuminate contradictions, tensions, and issues that are often invisible when traditional moral lenses are employed. In this way, Sevenhuijsen's work is of particular

\footnotetext{
${ }^{12}$ In chapter three, I present a more robust discussion of the differences between the ethics of care and other moral theories. For this reason, my discussion here is brief.
} 
relevance, given how it links the politicization of care ethics to implications for social policy evaluation and development (Mahon and Robinson 2011, 5).

\subsection{The Ethics of Care and Social Policy}

The most recent literature on the ethics of care echoes Sevenhuijsen's contribution and focuses on social policy. It also expands this focus to include transnational policies and governance, and impacts of globalization (Mahon and Robinson 2011, 10-11). In particular, the work of Fiona Robinson (1999; 2006a; 2006b; 2011a; 2011b; 2013b), Olena Hankivsky (2004; 2006; 2011) and Fiona Williams (2001; 2010; 2011) are notable examples of this scholarship.

Robinson argues that the ethics of care has particular implications within the context of social policies, globalization and transnationalization: care ethics focuses on practice, and

when 'doing' ethics is understood this way, it becomes clear that the moral theorist cannot choose to abstain from the work of really looking at social, economic, and political arrangements by claiming that they are not the stuff of moral theory. (Robinson 2011a, 135)

Furthermore, Robinson asserts that given the transnationalization of care, and the continual globalization of social, political, and economic arrangements, the ethics of care needs to be extended to issues such as the flow of social policy discourse and global care chains (Mahon and Robinson 2011, 10-11). Within this larger global context, Robinson suggests that:

Unlike a women's rights approach, a critical care ethics approach can help us to understand why women are economically and physically exploited and subject to violence through elucidating the connections between femininity and subservience, on the one hand, and masculinity and autonomy, on the other. (emphasis in original, 2011a, 144) 
Arguing that globalization has a 'masculine face,' supported by privatization and autonomy, Robinson suggests that a critical ethics of care can help unveil the hidden aspects of globalization, including the sexualized, racialized, class-based regime of lowwage caring services provided by mostly female migrant workers that is essential to the continuation of capitalist processes, and the morality that legitimizes such processes (Robinson 2011a, 138).

Hankivsky also sees the ethics of care as a valuable tool to guide us toward the creation of more ethical forms of globalization (2006). While acknowledging that there is still much work to be done in order to understand how care ethics can influence globalization in this way, Hankivsky uses three principles of care to suggest starting points and possibilities $(2004 ; 2006)$. Firstly, she argues that the principle of contextual sensitivity, central to the ethics of care, prioritizes a more encompassing approach "to understanding the harm and suffering associated with various forms of globalization" $(2006,99)$. Because the ethics of care is committed to understanding the contexts in which issues and oppression arise, Hankivsky asserts that it is better suited to analyze such concerns in a globalized world, which often involve multiple factors, scales, individuals, and different levels of state power (2004; 2006; 2011). Secondly, citing Robinson (1999), Hankivsky suggests that responsiveness, the ability to perceive others on their own terms, can translate into "a unique form of interaction... [which] has the potential to disrupt and counter the hegemonic forces that dominate current discourses and practices of globalization" $(2006,102)$. Finally, noting that care ethics is "concerned expressly with the actual outcomes and practical and material effects on people's lives of making certain choices and decisions" $(2004,38)$, Hankivsky argues that the ethics of 
care can assist in achieving the ultimate goal of promoting the well-being of all peoples while also accepting their autonomy and differences in a globalized world (2006, 104; 2011, 158).

Finally, much of Williams' work involves the analysis of policies, across various scales, from an ethics of care paradigm $(2001 ; 2010 ; 2011)$. She explores the tensions and contradictions that result in care policies from the perspective of both caregivers and care receivers (2010), and seeks to understand "what values and principles should underpin a new welfare strategy, and how it can be defended" (emphasis in original, 2001, 467). Again emphasizing the more complex links across micro- and macro- scales under transnationalization and globalization, Williams is highly concerned with how policies affect the individuals involved in caregiving and receiving. She writes:

[my] reason for asserting the importance of care as a political issue is that to talk about an 'ethics of care' may provide us with a more robust discussion of the values that are important to people in their relationships of care and intimacy. $(2001,475)$

Williams' work is also remarkable for its engagement with dis/ability literature.

While acknowledging that

for many, the very concept of 'care' embodies an oppressive history in which the practices and discourses of paid (particularly professional) and unpaid carers have maintained disabled and older people in a position of, at worst, unwanted dependency, abused and stripped of their dignity, and at best, patronised and protected from exercising agency over their lives $(2001,478)$,

Williams argues that there are still commonalities between recent conceptualizations of the ethics of care and those concerns raised by disabled activists. Although she does not address all of the tensions between these two perspectives, Williams work is noteworthy in its attempt to link these perspectives, particularly by emphasizing the distinction 
between independence as self-sufficiency (which she, along with Tronto (1993) and Sevenhuijsen (1998), rejects) and "independence as having the capacity to have choice and control over one's life" $(2001,480)$. She also highlights how despite the constructed binary between able-bodied and disabled, in practice, many people are both caregivers and care receivers at different times and in different situations; this, she argues, must be considered in any application of the ethics of care to policy. 


\section{Chapter: A Critical Ethics of Care}

In this chapter, I present my understanding of a critical ethics of care. I draw on the works of Tronto (1993) and Robinson $(1999,2011 b)$ in particular, and argue that assessments of social policies using a critical ethics of care will benefit greatly from a feminist political economy methodology (Robinson 1999, 129-31). Finally, I develop an argument to support my choice to use the ethics of care as an ethical framework from which to evaluate the Newfoundland Paid Family Caregiver Program, as opposed to other mainstream ethical approaches.

In the first section, I provide a theoretical discussion of care. This term is often used and seldom defined; however, in the specific context of care ethics, it is both useful and necessary to establish what is meant by 'care.' In section two, I build off of this definition of care, and explore the ontology and moral subject of the ethics of care. Significantly, the ontology and moral subjectivity of care ethics is relational; as such, in this section I also argue that a relational starting point more accurately reflects the material reality of the human life course. I then return to Tronto's work, and review her four criteria for the ethics of care in detail. These criteria are attentiveness, responsibility, competence, and responsiveness (1993); I interrogate each of these criteria in turn.

In section three, I argue that a truly critical ethics of care must explore the material realities in particular contexts and the social, political, and economic systems which comprise these realities. In order to facilitate this, I follow Robinson (1999) and argue that a feminist political economy methodology can be combined with care ethics to more effectively uncover the power relations and political-economic processes in which all moral decisions are made and in which all moral actions are executed. I demonstrate 
the value of this methodological approach by showing how a feminist political economy understanding of gender illuminates the ways social-political-economic systems and ideology permeate all things, including ethics. By critically uncovering the ways in which symbolic gender influences ethical framings, the potential for the ethics of care to address gender-based power imbalances is revealed. While highlighting only one example, I believe that this analysis demonstrates the benefits of linking feminist political economy approaches with the ethics of care, as together, these methodologies provide the tools necessary to place relations of care within systemic grids through which power and privilege are distributed.

Finally, in section four, I argue that normative analyses of social policy is important, and I present my reasoning for using a critical ethics of care as the ethical framework from which I investigate the Newfoundland Paid Family Caregiver Program. ${ }^{13}$ I do not compare and contrast the ethics of care to other specific ethical frameworks. Rather, I identify some principles common to a selection of some of the more prominent mainstream ethics, and illustrate how a reliance on an ethics which stem from such tenets risks perpetuating the marginalization of those who do not fit the mythical mould of the independent, self-sufficient, and autonomous moral subject. I further argue that these principles often obfuscate the role of political-economic systems and power dynamics in shaping our lives. In contrast, a critical ethics of care starts from very different premises which facilitate moral practices that do not (re)produce such marginalization by seeking to understand how power dynamics and privilege impede our

\footnotetext{
13 This is the subject matter of chapter four.
} 
ability to maintain caring relations for all. For this reason, I contend that the ethics of care avoids the pitfalls of traditional approaches to ethics and is thus a fruitful way to interrogate matters of morality, ranging from the moral issues we face in our everyday lives to the establishment of policy and political decision-making at the global scale.

\subsection{Theorizing Care}

It is not possible to develop the ethics of care without a theory of care (Sevenhuijsen 1998, 83). This task is not to be taken lightly; care literature is enormous in scope, and the basic concept in this literature - care - is widely contested (Day 2013; Hughes et al 2005; Phillips 2007). For instance, Harrington Meyer describes care as "moments of dependency, moments when we rely on the kindness or generosity of others to provide for our most basic needs" $(2000,1)$. By extension, care work is that labour which provides for the basic needs of others in such moments of dependency (Duffy 2011, 2). Armstrong and Braedley further argue that care, care work, caregiving, and care receiving are relational $(2013,10)$, while Phillips (2007) notes that care can be viewed as a commodity. These are just a sample of the many ways in which care is defined and described in this literature.

Care has also often been defined through generalized ideas about those who do care. Fisher and Tronto (1990) point out that rather than fully examining the practice and activity of care, discussions of care often focus on the actors (36). For example, 'the visible carer' arises from concerns about the devaluation of women's care work, and suggests that such devaluation can be counteracted by making this care work visible, while 'the androgynous carer' comes from the 'add men and stir' approach, and suggests that care work and caring itself will become valued and appreciated if men begin to also 
practice care work (Fisher and Tronto 1990, 35-36). This focus on the caring agent, rather than care as an activity, as Fisher and Tronto argue, comes from the fact that for one, we feel we already 'know' what care is - many of us give and receive care in a variety of ways on a daily basis - and for two, the legacy of the rational, autonomous $\operatorname{man}^{14}$ in Western philosophy leaves little space for discussing activities like care, which are centered on moments of dependency and complex relations (1990, 36).

In response to this tendency to focus on the actors, rather than the activity, Fisher and Tronto developed a definition of care which is now oft-cited (cf. Brandsen 2006, 206; Eckenwiler 2012, 20; Engster 2005, 50; Held 2006, 32; Herring 2014, 1; Sevenhuijsen 2000, 12; Sevenhuijsen et al 2006, 82). It is also the definition of care that I follow in this thesis:

On the most general level, we suggest that caring be viewed as a species activity that includes everything that we do to maintain, continue, and repair our 'world' so that we can live in it as well as possible. (emphasis in original, 1990, 40) ${ }^{15}$

I believe that this definition has many strong points. As previously discussed, this definition is clearly focused on the activity of caring; there is no mention of a particular caregiver. This is important because it avoids treating caregivers as a homogenous group (i.e. all caregivers are selfless, women, and so on). Furthermore, this definition allows for

\footnotetext{
${ }^{14}$ I discuss the rational, autonomous man in the third section of this chapter.

${ }^{15} \mathrm{I}$ wish to point out that this definition is similar to many definitions used to describe social reproduction. Social reproduction comes from the feminist historical materialist and Marxist traditions, and is generally defined as "as "the complex of activities and relations by which our life and labour are daily reconstituted" (Federici 2012, 5). In this way, I believe that care and social reproduction can be thought of as one and the same. However, I have chosen to use the term 'care,' as opposed to 'social reproduction' because social reproduction includes a focus on labour. While reproducing labour is, of course, important, I fear that if the focus is on reproducing labour, as opposed to reproducing a flourishing world in general, those who are thought of as 'unproductive' by mainstream economic discourse will be seen as less worthy of daily reconstitution. Given that this thesis is concerned mainly with the elderly population, this is of particular concern, as many of these individuals are retired or no longer work traditional jobs, and are thus viewed as outside of the 'productive' world which is so narrowly defined in neoclassical economics.
} 
space to include the many different culturally and contextually specific practices of care. Care, as a practice, varies across different contexts and relations, and care evolves through time. By broadly defining care as the entire spectrum of activities that contribute to the healthy (re)production of our world, specific practices of care are not excluded. For this reason, I believe that this definition avoids a western-centric view, in which care and caregivers are essentialized and homogenized according to specific Western ideals. ${ }^{16}$

Fisher and Tronto also expand on this definition by describing "four intertwining phases" $(1990,40)$ of care. Caring about is the component of the caring process in which we recognize that there is a need that should be met. Taking care of occurs when we take responsibility for meeting said need, while caregiving is the concrete work involved in addressing needs and maintaining a healthy world. Finally, care receiving involves the response to the caregiving by the person(s) toward whom the care is directed.

Significantly:

These phases are not orderly stages of caring which culminate in some completion of the caring activity. In practice, phases of the caring process may be intertwined in chaotic and contradictory styles: caregiving may proceed where no one any longer cares about the original situation. Or, the person who gives care may find herself also having to take care because of a vacuum of responsibility. Care receivers may fight caregivers about the kind of care being given. (Fisher and Tronto 1990, 41)

Thus, while it may be tempting to think of these components of care as a linear process, in reality care is messy, complex, and requires ongoing work.

An important point must be made here. Fisher and Tronto's definition, in my opinion, has sometimes been misrepresented in the literature. Notably, Sevenhuijsen

\footnotetext{
${ }^{16}$ See chapter two for a discussion of the essentialization of women and care, as well as western-centric understandings of women and care.
} 
describes the taking care of phase as 'caring for,' and calls the caregiving phase 'taking care of' $(1998,137)$. While this may appear to be a simple terminological difference, I am concerned with the misuse of the original terms because the terms Sevenhuijsen uses can refer to different understandings of care in the literature. For instance, Ward-Griffin, citing Ungerson (1990), divides caregiving roles into two components: caring about, which involves feelings of affection and love, and caring for, which involves the meeting of a variety of needs, ranging from physical, to emotional, to mental, to spiritual (2008, 5). In this case, caring has been divided into two categories: one involving emotional labour and one involving physical labour. This distinction, I believe, is problematic for several important reasons.

For one, the association of care with emotion resembles the ethics of care arguments in which women are essentialized as caregivers (à la Noddings). If emotions, and love in particular, are one of the components of care, care is once more easily relegated into the private sphere in which families, and often women specifically, are responsible for caregiving and receiving. As Tronto (1989) suggests, the distinction between caring for and caring about "fits with the engendered category of caring in our society" (174). Men care about, while women care for:

Caring about refers to less concrete objects; it is characterized by a more general form of commitment. Caring for implies a specific, particular object that is the focus of caring. (Tronto 1989, 173-4)

In other words, caring about is a masculine task: historically in our society, men have largely been responsible for 'caring about' social-political-economic issues, things which are not tangible (but nonetheless, greatly impact those which are). Women, on the other hand, are responsible for 'caring for,' completing the everyday (and unending) tasks that 
have often been compartmentalized as issues for the private home. Such binary understanding of care is too simplistic to capture fully the very complicated reality of caregiving, care receiving, and care work. It seems to suggest that care can be divided into isolated tasks; however, care and all the components of care are intricately connected, non-linear, and very much dependent upon each other. Perhaps even more concerning, this binary of caring about/caring for reflects symbolic constructions of gender ${ }^{17}$ which have been used to value men's work while simultaneously devaluing women's work, thereby (re)enforcing the devaluation of women more generally.

The association of care with love is also very limiting. While love can often be a powerful motivator to care, it cannot be a defining characteristic of care if care is to have any political meaning. For instance, how can one argue for an ethics of care that can be used to evaluate and assess moral practices related to people in distant lands if, in order to 'care,' we need to feel love? Is it possible to love people you have never met? Heading down this road is a dangerous one, and relying on emotions to define care, I believe, obscures the reality of caring, the reality that all of us depend on care and give care every day, and the reality that caring relations are necessary to the flourishing of our world as a whole. $^{18}$

Secondly, as I have argued elsewhere, dividing care into categories such as emotional versus physical labour can lead to a situation whereby certain types of care are

\footnotetext{
${ }^{17}$ See section three of this chapter.

${ }^{18}$ Importantly, this is not to suggest that the ethics of care means that we can or should 'care' about people in distant lands either. Rather, it is to suggest that if we choose to engage in global issues, then the realities of care in that particular context need to be carefully interrogated. Thus, care, as in the material reality of care, can form the basis of a meaningful ethic from which to guide international relations, while love, I believe, is problematic and limiting in such contexts.
} 
privileged over other types of care (FitzGerald Murphy 2014). In other words, focusing on emotional versus physical caregiving may produce a hierarchy of care. To demonstrate, consider two other major conceptual frameworks for understanding care: nurturance and reproduction. As Duffy (2005) shows, the fault line between nurturance and reproduction can actually hide important differences in care work, and the people who do said care work. Nurturance is described by Duffy as "a process or practice that has a strong emotional dimension and is based on human connection in relationship" $(2005,68)$, whereas "reproductive labour is defined as work that is necessary to ensure the daily maintenance and ongoing reproduction of the labour force" $(2005,70)$, including "a whole range of activities, some of which are relational, and others of which - such as shopping and doing dishes - are not" $(2005,69)$. As Duffy shows, these two types of work are also often racialized, with women of colour performing the majority of reproductive labour, while white women do nurturing work. By focusing on one of these frameworks over the other, certain individuals are therefore excluded from the analysis while others are not: "by focusing on framing care as nurturance, scholars may obscure one of the major axes along which the racial division of reproductive labour has historically occurred" $(2005,80)$. I argue that similar to the nurturance/reproductive division, a division between physical care labour and emotional care labour also risks marginalizing certain groups of individuals who perform 'more' of one type of labour than the other.

Tronto and Fisher provide an excellent example which demonstrates this in their article "Toward a Feminist Theory of Caring" (1990); this example is worth quoting at length here. During a discussion of care in a women's studies class on mothering, the 
authors' note, the notion of caring described by many of the students resembled the 'cult of domesticity' (cf. Cott 1977). The cult of domesticity is a term which describes the myth of the white, middleclass woman whose entire being is defined by her duty to care, particularly in emotional ways, in the private sphere of the home while her white, middleclass husband exchanges his labour for a wage in the capitalist marketplace. As the author's note, despite the fact that this image was a myth for many of the women in the class, caring was seen as valuable through this lens and this lens alone. Significantly, besides being an inaccurate reflection of their own experiences, this ideal also marginalized the student in the class who was not white:

During this entire discussion, Diane, the one black woman in the women's studies class, has been holding back her anger. Finally she bursts forth. Her mother, a divorced woman who worked as a night nurse to support her children, had had little time for long discussions about their emotional realities. Her talks with them took place during the chores they performed together or even during her daytime rest period, when she allowed the children to wake her to tell her about things important to their lives. Much of her caretaking revolved around making sure her children were safe and well while she worked - finding friends and neighbors to look after them or taking them along to the hospital where she tucked them into extra beds. Each morning when she returned home, she would cook a pot of food for her children to eat when they got home from school. That flavorful pot, those nights tucked in the hospital beds, those calls to friends and neighbors - all these things mean 'nurturance' in Diane's life. (Fisher and Tronto 1990, 38-9)

The way in which care is (narrowly) defined can often mean that those who did not/do not experience care in that particular way are marginalized. At the same time, the care that they $\mathrm{did} / \mathrm{do}$ experience, because it does not fit neatly into the definition of care used in that context, is effectively rendered invisible.

This example illustrates one of the reasons I choose to follow Fisher and Tronto's definition of care. As discussed above, Fisher and Tronto developed their definition because they wished to focus on the activity of care, as opposed to the actors. However, 
as Duffy effectively demonstrates, the definition of the activity that comprises care can, and often does, have far-reaching consequences for the people that do care. Fisher and Tronto's definition, I believe, is wide enough to encompass all manner of specific caring about, taking care of, and caregiving/receiving practices, including nurturant and nonnurturant activities (Duffy 2011), and emotional and physical care. It leaves space to account for the differences that arise when caring for dependents, versus caring for superiors or caring in symmetrical relations (Waerness 1996). It need not privilege one type of care activity above another, and does not rely on culturally or temporally specific notions of care. Instead, this definition of care emphasizes the final outcome of care, the (re)production of healthy relations and human life more generally. Any activities that contribute to this task can thus be rightfully acknowledged and valued, as can the people that perform such tasks.

As a final note, I follow Armstrong and Braedley (2013) in that I assert that care should be a collective, not individual, responsibility. This is not to suggest that care cannot, in specific contexts, be provided individually. Rather, this is to make the normative assumption that there is a collective responsibility "to ensure that appropriate care is provided when needed and that failures of care are addressed" (Armstrong and Braedley 2013, 10). As Williams notes, “contemporary social processes of care are multirelational" $(2001,470)$, and care relations are influenced by all other systems of relations, power structures, and social, cultural, and economic processes. Because of this, care cannot be designated to the private sphere. While specific moments of caring about, taking care of, caregiving, and care receiving may take place between individuals, all of these moments and acts are, in some way, shaped by larger social and collective 
structures and processes. To assert, then, that care is an individual concern, is to ignore the fundamental interconnectedness of all our relations, to neglect the fact that "care is central to the construction of a functioning society, and that it is the source of societal empowerment and fulfillment” (Daly 2013, 37).

\subsection{Critical Ethics of Care}

A critical ethics of care, which arises from the realities of caregiving and care receiving that shape and inform all of our lives, cannot be reduced to simple logic statements and generalized rules, whereby one person's moral actions in one situation can be universalized and extrapolated as moral across all situations. Thus, unlike many other ethics, care ethics cannot be neatly summarized as a list of abstract principles (Robinson 1999, 40). Instead, the ethics of care is characterized by certain tenets which provide the skeleton from which to conduct moral reasoning without compromising the fact that all situations and actions are unique, and as such, all ethical actions must consider this uniqueness.

\subsubsection{A Contextual Moral Theory}

An ethic starting from care must be what Tronto calls a contextual moral theory:

All contextual moral theories share a sense... that more is necessary to describe morality than the delineation of moral rules and the requirement that humans will use their reason to understand and to apply these moral rules. Contextual theories may require as well as such principles a number of other human moral qualities. $(1993,27)$

The ethics of care is deeply concerned with context, and any moral guidelines stemming from the ethics of care must take into account the specific and material particulars that shape and inform our lives. The literature on care from political and human geography is 
helpful to understand this. Milligan and Wiles refer to 'landscapes of care' as a way to articulate care

through the differing, and sometimes surprising, social spaces that enable caring interactions; as individual space-time trajectories through varied social landscapes of care, care-giving roles, employment and social policies, and gendered and generational expectations of care and work; through the entanglements of exclusion and inclusion, dependency and independency that can manifest within and across formal and informal spaces of care; and through the emotional landscapes that underpin care and care interactions. Such landscapes can encompass the institutional, the domestic, the familial, the community, the public, the voluntary and the private as well as transitions within and between them..., [and] requires an understanding of macro-level governance or social arrangements that can operate at either (or both) the national or international scales as well as the interpersonal. $(2010,738)$

These landscapes of care, ranging from the physical terrain to the micro- and macrosocial relations in which we are located to the ideologies that shape our lives, are as varied as the particular lives and spaces individuals occupy. An ethic starting from care thereby challenges contemporary moral theories which rely on universal rules and principles to guide actions, simply because this ethic understands and acknowledges the particularity of care (Tronto 1989). It explodes the myth that we can easily transition between 'ought and is' (Robinson 1999, 1), between universal rules and our actual material reality, and suggests a different kind of ethics which starts from a critical interrogation of our particular and unique positions within complex landscapes of care, and which understands that our lives "find meaning only within webs of personal and social relationships" (Robinson 1999, 6).

\subsubsection{Ontology and the Moral Subject}

The ethics of care stems from a relational ontology.

It starts from the premise that people live in and perceive the world within social relationships; moreover, this approach recognizes that these relationships are both 
a source of moral motivation and moral responsiveness and a basis for the construction and expression of power and knowledge. (Robinson 1999, 2)

We are all (re)produced and sustained by our relations. While this statement tends to conjure images of personal relations, such as those between family and friends, it is crucially important to understand that such relations constitute only one part of the relational webs within which we are all intertwined. Our individual identities, needs, and realities are shaped "through their intersection with a range of private and public social and institutional arrangements" (Hankivsky 2004, 34). This includes our interpersonal interactions, but also the social, political, economic, and cultural systems which inform our lives. The complex webs of relations that (re)produce and sustain all of our lives create the world in which with live; our lives are defined by these social constructions rather than supposed 'natural laws' (Robinson 1999, 41). In this way, the ethics of care also acts as a lens from which to understand politics and power dynamics. The ethics of care prioritizes the relational reality that shapes all people's lives while acknowledging that care, politics, and ethics are intertwined.

The concept of the moral subject in ethical approaches is meant to depict human nature (Sevenhuijsen 1998, 55). When an ethical approach starts from a relational ontology, as described above, the moral subject is also characterized by relations. In this way, the moral subject in the ethics of care is a 'relational self' (Hekman 1995, 73), a self which only exists in relation to others at the most fundamental level (Robinson 2011). This moral subject is not an individualized, atomistic, pleasure-seeking being, but rather is already-always connected to individual and personal relations, political, economic and social systems and structures, and landscapes of care in all their varied forms. 


\subsubsection{Tronto's Four Criteria}

Care ethics, as previously reviewed, is fundamentally based on a relational ontology and is highly concerned with context. In Moral Boundaries, Tronto further elaborates on care ethics and identifies a more explicit description of the ethics of care. She explains: "to be a morally good person requires, among other things, that a person strives to meet the demands of caring that present themselves in his or her life" (1993, 126). While this initial statement is, as Tronto acknowledges, vague, Tronto uses it to demonstrate the practical nature of the ethics of care:

In the first place, the language of striving suggests that the moral aspect of care does not necessarily turn upon the success or failure of caring. In the second place, the language "as it presents itself" seems to permit actors to escape from their failures to acknowledge needs for care. These qualities spring from the fact that the ethic of care is a practice, rather than a set of rules or principles. (1993, 126)

To further round out this definition, Tronto also provides four moral qualities or elements of the ethics of care. These qualities are attentiveness, responsibility, competence, and responsiveness; I review each of these in turn below. Before doing so, however, I must be clear that not all care ethicists use these four elements. For instance, Hankivsky (2004) discusses contextual sensitivity, responsiveness and consequence of choice while others do not use specific elements in an explicit fashion (cf. Sevenhuijsen 1998). I have chosen to draw upon Tronto's elements, however, for three reasons. Firstly, I feel that her four elements encapsulate the elements used by care ethicists who do use such elements, even if they are labeled differently. For instance, I suggest that Hankivsky's contextual sensitivity can fall under Tronto's attentiveness criteria, while responsiveness and consequence of choice are strongly linked to Tronto's competence and responsiveness 
criteria. Secondly, Tronto's four criteria correspond to the four phases of caring (Fisher and Tronto 1990) discussed in the preceding section. By linking these elements of the ethics of care to the theory of care, I assert that these elements further reinforce the fact that the ethics of care arises from the lived realities of care and care relations.

Lastly, I see the ethics of care as a microscope; it provides a critical lens from which to examine and understand the world around us starting from the reality that care sustains and (re)produces us all. Like the knobs on a microscope, each of these criteria can be used to bring into focus different facets of the object of study. By using each criterion, and turning the knobs of the microscope, the ethics of care can uncover dimensions of the object of study that would otherwise remain hidden; uncovering such dimensions is of the utmost importance when trying to investigate and assess moral dilemmas and issues. Significantly, it must be emphasized that, as Tronto notes, while each of these elements can be considered individually, the integrity of the ethics of care relies on the fact that these elements are intertwined and interacting in any care relationship; the microscope requires all of the knobs to function properly and reveal the underlying microbes.

\section{Attentiveness}

The element of attentiveness, according to Tronto, involves the identification and recognition of a need, or indeed, needs $(1993,127)$. "If we are not attentive to the needs of others, then we cannot possibly address those needs" (Tronto 1993, 127). This criterion goes hand in hand with Fisher and Tronto's first phase of caring, caring about. One cannot care about something if one cannot recognize or identify its needs. 
While this may seem like a basic concept, as Klaver and Baart (2011) note, attentiveness has several nuanced meanings. For instance, in everyday language, being attentive can imply watchfulness, or cognitive processes like listening and concentration, as in, 'a student must be attentive in class.' In this context, attentiveness need not involve the identification of needs. In the context of care, however, attentiveness must extend beyond simple consciousness; it involves the quality of individuals to open themselves up to the particular and specific needs of others. As Hankivsky suggests, this is congruent with contextual sensitivity: "the basic knowledge of an individual requires full comprehension of that person's particularity" $(2004,32)$.

It is also crucial to note that while this may be the 'first' criterion in Tronto's formulation of care ethics, in no way does this suggest that attentiveness, once a need is recognized, ends. Rather, attentiveness requires ongoing work. As Tronto points out, in today's society we have an unparalleled ability to learn of the particular and material realities of people around the globe. However, the temptation to ignore the oppression, violence, and injustices we learn of is immense, even when physical distance is not an issue. True attentiveness requires an ongoing commitment to contextual sensitivity, and an understanding that 'others' matter. When the ethics of care is taken seriously, ignorance becomes a moral failing (Tronto 1993), and while there is a distinction between purposefully ignoring needs and simply being unaware of them, the ethics of care shows us that we must work to avoid ignorance, whether it be intentional or not. For this reason, as Tronto writes, "attentiveness, simply recognizing the needs of those around us [or indeed, the needs of those who may be far away], is a difficult task, and indeed, a moral achievement" $(1993,127)$. 


\section{Responsibility}

When the second phase of care, taking care of, is considered, responsibility becomes a moral category of great importance. First and foremost, I wish to distinguish responsibility from 'obligation' and 'duty.' Unfortunately, the literal definitions of these words are not helpful in this task. Responsibility, according to the Oxford English Dictionary, is the 'capability of fulfilling an obligation or duty,' while obligation is 'the action of constraining oneself by oath, promise, or contract to a particular course of action,' often applied in a legal sense. As such, responsibility appears to be a subset of obligation, and obligation is a consciously chosen contract in which one dedicates oneself to a particular course of action. Furthermore, the use of the word 'capability' tends to imply an individual's ability. That is, if you are not able to meet your obligation, then you are not capable, and this is an individual failing on your part. This understanding of responsibility, I believe, does not align with the ethics of care, which understands that a person's 'capability' is informed by their particular positionality.

As opposed to this understanding of responsibility, I suggest that responsibility is a cultural and social practice, it is embedded in our social relations and it is not a set of rules, nor is it always freely chosen. This distinction is subtle, but important. Robinson demonstrates it well in her discussion of responsibility and the state:

$[\ldots]$ the question for normative theorists are not if these states can take responsibility for their peoples, or what agent can act when faced with a crisis, or even what agent should be assigned the responsibilities/duties to prevent a crisis; rather, they should be, How are existing responsibilities within a nation-state distributed, and how are these responsibilities accounted for or justified through norms and social practices?

Understood in this way, responsibility in world politics is not synonymous with 'duty;' rather, it is part of everyday social relations... responsibility cannot be 
seen only as something that can be assigned after ethical deliberation; rather, it is central to what morality always already is. (emphasis in original, 2006b, 170)

While Robinson is speaking directly about responsibility and the state, the general idea can be extended to all peoples, all social structures, and all systems. Responsibility is always present, and there are individuals, collectives, systems, and relations which always-already take responsibility for the fulfillment of certain needs (or alternatively, do not take responsibility for said needs ${ }^{19}$ ). From an ethics of care perspective, the question of responsibility is not simply about 'assigning' tasks, fulfilling obligations, or freely choosing to perform certain duties. Indeed, such an understanding of responsibility can be harmful, as it leaves space for conflict if some individuals or groups feel they are (unfairly) obligated to address certain needs, and totally obscures the fact that often times, responsibilities are not freely chosen. As Hankivsky writes:

Sometimes caregivers undertake and continue their work out of a sense of forced obligation or even fear. As Hilary Graham argues, 'caring... is experienced as a labour of love in which the labour must continue even when the love falters'. (2004, 117; quoting Graham, 1993, 16)

Instead, the understanding of responsibility that I describe here emphasizes the fact that responsibilities, which are woven into the fabric of our lives, are distributed. The question, then, becomes how are responsibilities distributed, and how do the existing political-economic systems, cultural practices, and power dynamics affect this distribution?

\footnotetext{
${ }^{19}$ For example, Tronto (2013) argues that certain kinds of activities earn 'passes' from care work. Individuals involved in protection and in control of protective work earn a "'protection pass' out of responsibility for other, more feminized forms of care work" (79) while 'productive passes' are earned when people (generally men) work in the market (82). Tronto further discusses how the distribution of such passes follows gendered lines: “[...] what it means to be masculine is to be given a 'pass' out of thinking about 'girl things,' including the caring responsibilities assigned to women" (68).
} 
Notably, this discussion of the distribution of responsibilities is distinct from the framework developed by Engster (2005), based upon Robert Goodin's assigned responsibility model of moral obligation $(1985 ; 1995)$. Goodin suggests that we all have a moral responsibility to protect the vulnerable, but that generally, protecting the vulnerable is most effectively done by particular individuals. Thus, this model implicitly argues that the responsibility of care is, and should be, based on intimate relations. Engster explains:

[...] Goodin argues that we are justified in showing partiality toward our special dependents on the grounds that assigning responsibility in this particular way generally means that all vulnerable individuals will be more effectively and excellently protected. An important correlate of this model, however, is that we all become responsible for protecting vulnerable individuals if the persons appointed to look after them should fail to perform their assigned duty. $(2005,66)$

Engster suggests that this model can be applied to caring relations. He argues that based on this model, our primary duties to care may be said to be owed to ourselves and to those with whom we have a special and particular personal relationship to, or are otherwise specially oriented and able to help $(2005,66)$. Those with whom we are in a special position to help include those with whom we have an intimate relationship or social relationship. Clement describes a similar model, also based upon Goodin's work:

First, our care obligations within the private sphere are based on our friends' and family members' vulnerabilities to us. Second, people beyond our private sphere are also vulnerable to our actions and choices, and thus we also have care obligations to them. (emphasis in original, 1996, 75; also quoted in Robinson 1999, 28)

While I agree that in some instances, 'special personal relations' facilitate the provision of care, I disagree with this understanding of the distribution of caring responsibilities. To argue for a distribution of caring responsibilities (largely) based on personal relations is 
to ignore the ways in which the larger landscapes of care shape the ways that we can, or cannot, provide care. It ignores the fact that individual personal relations are enmeshed in larger webs of social-political-economic relations, and that these webs thereby inform (and are informed by) said personal relations. To put this succinctly, this approach means that the larger context in which caring takes place is ignored. By ignoring such context, this framing resorts back to a 'universal' ethics approach, in which the 'rule' or guiding principle for all scenarios is generally that proximity is the best way to distribute caring responsibilities. Such a concession, in which abstract rules determine our responsibilities, totally negates the ethics of care, which resists abstract rules and seeks to understand the ways in which our particular positions influence the (re)production of healthy and fulfilling lives. For this reason, I reject such a conceptualization of responsibility and instead, following Robinson, understand responsibility to be directly linked to the issue of contextual sensitivity, and to the fact that we are all related in complex and far reaching ways. Responsibility is informed by the particular care landscape in which needs arise; understanding this landscape and how responsibility is shaped and distributed by said landscape is the central goal of this particular criterion.

\section{Competence}

Competency corresponds to the third phase of caring, caregiving. Tronto writes:

To include competence as a part of the moral quality of care, is obviously to align this approach with moral consequentialism. Intending to provide care, even accepting responsibility for it, but then failing to provide good care, means that in the end the need for care is not met. $(1993,133)$

This criterion can be controversial (Tronto 1993, 133), as it is possible for it to be interpreted as a critique of well-meaning individuals or groups who attempt to meet the 
needs of others but, however measured, 'fail' to do so effectively. Regardless of intention, though, the consequences of care are of the utmost importance. The ethics of care, as I have argued throughout this chapter, "is concerned expressly with the actual outcomes and practical and material effects on people's lives of making certain choices and decisions" (Hankivsky 2004, 38). If proper attention is not paid to such actual outcomes, the very foundation of the ethics of care as a moral practice is violated.

A thought exercise can help to better understand the importance of this criterion. If we consider a nurse who, because of inadequate healthcare funding, is unable to do their job effectively, is it correct to morally condemn this person for failing to properly provide care? It is natural for us to become squeamish at the thought of doing so - surely the nurse cannot be held accountable for a lack of resources? At the same time, however, if we do not constantly evaluate whether or not competent care is being provided, then it becomes possible that the provision of good care is impossible, and those involved in such scenarios become absolved from responsibility of meeting the caring needs.

This is not to suggest that we individually blame the nurse. Instead, we must once again rely on a contextual analysis to attempt to understand the many dynamics which are causing the incompetent care. The fact that the nurse is not providing competent care is a signal, and an important one at that, that somewhere within the complex web of social relations, there is a failure to meet the caring needs. If we shrug off this signal, and say that the nurse cannot be responsible for the poor care because of exogenous variables, we miss an important clue and opportunity to (re)assess the larger caring landscape in which the nurse is working. We are therefore once again reminded of the importance of contextual sensitivity. While it is necessary to hold each care relation, from those 
between individuals to those between nations, up to a certain standard, it is also imperative to evaluate continually the material and particular factors that are shaping the care relation. Competency is an effective way to evaluate the care relation, albeit only when the care relation is placed within the larger context of care. The criterion of competency ultimately reminds us that we need to constantly be aware of how decisions and choices affect the lives of real people.

Responsiveness

Responsiveness, which Tronto says "signals an important moral problem within care: by its nature, care is concerned with conditions of vulnerability and inequality" $(1993,134)$, is meant to not only highlight the unequal power relations often involved in caregiving and care receiving, but also to indicate that the care receiver should have agency in this relation $(1993,135)$. For this reason, it corresponds to the fourth phase of care, that of care receiving.

There are some nuanced points that must be made when considering responsiveness. As has been argued throughout this piece, caring relations involve, to at least some degree, vulnerability and dependency. However, the responsiveness criterion reminds us that even though caring relations involve dependency, this is not to suggest that the care receiver is wholly and completely dependent on the caregiver. Instead, I suggest that the responsiveness criterion facilitates an understanding that care and dependency are not antithetical to agency; rather, the question that must be examined is how inequalities, which often arise through systems of oppression, domination, and 
exploitation, affect our ability to maintain agency and autonomy ${ }^{20}$ in our ongoing dependencies. Responsiveness creates space to check continually that our caring relations, which are embedded in structures of inequality, do not become abusive, paternalistic, or colonial, ${ }^{21}$ and ensures that we take seriously the perspectives of all people, but particularly those who experience inequality, which thereby impacts their relations of care.

Finally, it is important to note here that disability literature often contests the use of terminology like 'dependency,' which is understandable in the context of disability studies, where 'care' has often been intricately intertwined with oppression for peoples with disability (cf. Kröger 2009; Williams 2009). Daly claims, in fact, that in disability rights theory, care is equated with dependency $(2013,34)$. However, as Milligan and Wiles point out, such arguments may be a consequence of how we theorize care itself $(2010,737)$. That is, when care is seen as unidirectional, and when care receivers are seen as passive, then abusive 'care' is certainly a possible consequence, and the research which exposes such 'care' is important. However, in my own use of the term care, as described above, I see care as necessarily relational. Part of that relation is that even if one person is dependent, they still have space to respond to the relation, and indeed to contribute to the relation itself. Dependency, as I theorize it, is not a negative thing; it is a reality of all our lives. I reject the universal equation of dependency and oppression, and assert that the real issue lies in the inequalities that exist through various oppressive systems and power dynamics which create the possibility for certain relations of

\footnotetext{
${ }^{20}$ Importantly, as Hankivsky writes, "unlike autonomy as conceptualized in the justice tradition, we are talking about an autonomy that prioritizes relationships, integrity, and self-respect" $(2004,120)$.

${ }^{21}$ For a discussion of colonialism and care relations, see Narayan (1995).
} 
dependency to become misused and abused. The criterion of responsiveness creates space to interrogate such inequalities by advocating that all agents in the caring relation must be able to respond, in an ongoing manner, to the caring relation.

\subsection{Methodological Approach: The Ethics of Care and Feminist Political Economy}

The ethics of care starts from the premise that context matters. Such a presupposition requires that we "consider the ways in which arrangements for care are influenced by and influence historical, political, economic and social relations" (Armstrong and Braedley 2013, 11). As scholars like Daly (2013) and Robinson (1999) suggest, a critical ethics of care which draws upon a feminist political economy methodology can better illuminate such things by helping to uncover how care and care relations are filtered through other relations, systems, and power dynamics.

Feminist political economy is a methodology which focuses on critically uncovering and analyzing the structures and relations that create, reflect and sustain inequalities (Armstrong and Connelly 1999) while acknowledging the material differences and experiences of particular women (Luxton 2006). It is highly concerned with examining and understanding the ways in which political and economic systems shape the lives of real, living human beings; in other words, feminist political economy places the "personal experiences of individual women [and people in general] in a larger social and economic framework" (Luxton 1980, 13). Rather than relying on abstractions, feminist political economy privileges the lived realities of all people, and seeks to illuminate the complex ways social policies and care relations are intertwined. As the ethics of care is also concerned with material relations of care, and with understanding how these relations are informed by other social structures and relations, I believe that the 
ethics of care and feminist political economy can form a fruitful partnership as a critical methodological tool. As Hankivsky points out:

Care theorists have pointed out that, without some checks upon the care ethic, pathological forms of care can emerge. These include maternalism, parochialism, and colonialism. Without doubt, any viable theory of care must take into account the oppressive conditions under which caring practices take place. $(2004,22)$

Feminist political economy, I contend, can help care ethicists take such material considerations into account. To demonstrate the potential power of this union, I provide a feminist political economy reading of the moral subject in traditional ethics and care ethics so as to demonstrate how cultural, social, political, and economic conditions have a very real influence on the ways in which morality resonates in the particular and actual lives of both men and women. Through this example, I show how feminist political economy can uncover the complicated and often reified ways that larger systems of oppression, domination, and exploitation permeate the particular lives of real people; such uncovering, I believe is a crucial part of moral practice under the ethics of care framework. To begin this example, I start with a discussion of gender.

As feminist political economist Spike Peterson explains, "gender is a systemic social construction that dichotomizes not only men - women but also identities, behaviors, and expectations as masculine - feminine" (emphasis in original, 2003, 31). This constructed (and therefore mutable) binary

is a socially imposed and internalized 'lens' through which individuals conceptualize and perceive themselves and the world... [while simultaneously] the pervasiveness of gendered meanings [also] shapes concepts, practices, identities, and institutions in patterned (but not fixed) ways. $(2003,31)$

Tickner elaborates that gender, in the symbolic sense, is 
a set of variable but socially and culturally constructed characteristics - such as power, autonomy, rationality, and public - that are stereotypically associated with masculinity. Their opposites - weakness, dependence, emotion, and private - are associated with femininity. $(1997,614)$

Jennings (1993) provides a list which further demonstrates how this masculine -

feminine binary is used to define, characterize, and categorize a variety of things; part of this list is reproduced below:

$$
\begin{gathered}
\text { masculine / feminine } \\
\text { (market) economy / family } \\
\text { man / woman } \\
\text { rational / emotional } \\
\text { objective / subjective } \\
\text { competitive / nurturant } \\
\text { independent / dependent } \\
\text { efficiency / inefficiency }
\end{gathered}
$$

A feminist political economy perspective suggests that this symbolic system of gendering pervades and colours other relations. Morality, ethics, and the moral subject are not immune to this gendering, and like all social processes, practices, and institutions that reflect historical constructions of 'masculinity' and 'femininity,' the moral subject in much of Western thought has been, and continues to be, assigned and defined by a constructed notion of masculinity. While there are variations in the details of how this masculinity shapes the moral subject of Western thought, certain characteristics are pervasive; in particular, this moral subject is consistently defined as an independent, autonomous man.

The fact that this moral subject is a 'man' is no coincidence. This moral subject echoes the hegemonic masculinity (Connell 2005) perpetuated by binary gender systems. On this view, the man is tough, independent, rational, and detached; he is indeed the 
antithesis of care itself. The autonomous, independent moral subject is perhaps best characterized by homo economicus, or Economic Man. Williams writes:

Homo economicus is the offspring of a separative worldview that understands the nonself and self as totally independent... Events, things, other human beings, and nature exist apart from the self; they are to be acted upon, controlled, and/or measured. $(1993,146)$

Economic man is independent, autonomous, and rational. He is free to choose all of his actions without systemic constraint, and indeed, free to fully create his own identity and status. Of course, economic man is certainly not the only manifestation of this moral subject. Peterson and Runyan note that the concept of 'Political Actor' - "the legitimate wielder of society's power" - is not gender neutral, but instead, "effectively and sometimes explicitly exclude[s] women from definitions of 'human,' 'moral agent,' 'rational actor' and 'political man"' (1993, 34; also cited in Held 2006, 155). Marchand and Runyan refer to "Globalization Man," $(2011,17)$ another moral subject derived from hegemonic masculinity.

When economic man (or indeed any of his macho-cousins) is the supposed normative image which moral people strive to emulate, dependency is seen as an undesirable state. To be dependent means to be weak, incomplete, and flawed in some way. Yet, as Feder Kittay correctly points out, independence is a myth:

Independence, except in some particular actions and functions, is a fiction, regardless of our disabilities, and the pernicious effects of this fiction are encouraged when we hide the ways in which our needs are met in relations of dependencies. On the other hand, this fiction turns those whose dependence cannot be masked into pariahs, or makes them objects of disdain or pity. It causes us to refuse assistance when it is needed. It encourages us to either deny that assistance to others when they require it or to be givers of care because we fear having to receive care ourselves. $(2002,268)$ 
We are all dependent. Although this dependency takes different forms contingent on the particular landscapes of care we find ourselves in at particular times throughout the life course, the "independent unembodied subject who represents the ethical subject and the political subject or citizen" (Feder Kittay, Jennings, and Wasunna 2005, 445) cannot, and will not, be found. This, I assert, is the most radical, and indeed important, reason why the ethics of care must be taken seriously.

When the moral subject is understood to be embedded and shaped by relations of care, to be dependent and interdependent, the objectives and central concerns of moral philosophy are transformed. Instead of attempting to understand and obey abstract moral principles, morality is now centered on questions such as "how can I best express my caring responsibility?" (Tronto 1993); how can I foster relations which flourish and bring well-being? Significantly, as Sevenhuijsen points out, to pose these questions in the first person by no means suggests that an ethics guided by such questions is individualistic or private $(1998,57)$; indeed, as has already been discussed, relations of dependency and care are found across all landscapes of care, from the macro-political economy to the familial scale. Instead, it is to emphasize that the practice of ethics must focus on the answers to such questions. Ethics is no longer about a set of abstract rules which can be applied to various situations; instead, ethics is the task of understanding and addressing caring needs as they emerge and change through time-space. Care ethics moves ethics in general from the abstract realm to the material reality in which we all live, and in so doing, changes ethics from rules and theories to practice. For this reason, the ethics of care can be understood as a "concrete activity, in the sense of caring about and for daily needs; and as a moral orientation - as an ethics of a set of values that can guide human 
agency in a variety of social fields" (Sevenhuijsen 2000, 6; also quoted in Robinson 2006b, 164).

Finally, it is important to note that the ethics of care is not meant to provide a feminine replacement for the masculine morality/moral subject (Sevenhuijsen 1998, 15). In contrast, the ethics of care is a feminist ethic. This distinction is immensely important. A feminine alternative to economic man would only serve to further exacerbate the constructed binary between male - female; it would bring with it the "burden of accepting traditional gender divisions in a society that devalues what women do" (Tronto $1989,185)$. Such an ethic would be antithetical to the emancipatory goals of feminism. Instead, as Hoagland writes:

A truly radical ethics [must] challenge not only the masculine but also the feminine, for the feminine is born of a masculinist framework and so does not, at a deep level, represent any change. (1991, 259; also quoted in Hekman 1995, 66)

The ethics of care, I contend, has such radical potential. The moral subject in the ethics of care is not a romanticized, naturally, and essentially caring being. Rather, it is one who is embedded in landscapes of care, who shapes and is shaped by relations across all scales, and who is fundamentally dependent on other people, systems, and environments. This moral subject is not feminine, as characterized by the binary social construction of femininity. This moral subject is one that can only be thought of in relation to everything else. The ethics of care not only allows for the particular positionalities of each person to be accounted for, it demands it. As such, people are not measured against a masculine or feminine moral subject, nor are they pigeonholed into either category. Instead, people, and their caring needs, are assessed based on their own unique, particular, and specific positionalities. 
By drawing upon the feminist political economy understanding of gender, it also becomes clear how the moral subject, when characterized by masculine or feminine notions of gender, and morality and ethics based on such a subject, resonates in material ways. Historically, the (Western) masculine construction of morality has been oppressive for women. Tronto refers to this oppression in relation to three 'moral boundaries:' the boundary between morality and politics, the 'moral point of view boundary,' and the public/private boundary $(1993,10)$. Specifically, the moral-political boundary arises from the conception that morality and political concerns occur in two different spheres, morality is the domain of the feminine, while political concerns belongs to men. The moral point of view boundary stems from the idea that moral judgments must be objective; as Jennings' list above demonstrates, objectivity is masculine and thus women are excludable from this. Finally, the public/private boundary is a well known and often discussed issue in feminist political economy, and has been used to relegate women to the home and 'private sphere' while men are able to partake in the public realm (which is integral to the (re)production of the social relations of capitalism).

While the autonomous, rational moral subject has certainly contributed to the establishment and reproduction of these boundaries, the binary notion of gender that is critiqued by feminist political economists is a two way street. This is important, because it reminds us that focusing on the ethics of care as a 'women's morality' is counterproductive, and will only serve to reinforce these boundaries, and the binary thinking from which such boundaries sprang. Only by moving away from the idea that the ethics of care is somehow specifically female (and indeed the idea that care itself is specifically female (Sevenhuijsen 1998, 18)), can care ethics be truly political. Feminist 
political economy, as this discussion has shown, interrogates the ways in which gendering processes inform the material world and seeks to uncover how systemic oppression and power dynamics influence the particular lives of all people. For this reason, feminist political economy can assist in the task of politicizing care ethics by providing additional tools to uncover critically the ways in which care and caring relations are informed, structured, and shaped by binary gender constructs and political inequalities.

To conclude, I have argued that the ethics of care is a radical feminist ethics, ${ }^{22}$ as it recognizes that people occupy different spaces, have different privileges, and are affected by power in different ways. It indicates that all moral action must begin by understanding how these differences affect the lived realities of all people, and how systems of oppression, domination, exploitation, and other power dynamics, affect our ability to create caring relations that allow all peoples to flourish. Feminist political economy is a useful methodological tool to assist in this task of locating moral dilemmas and practice within these systems. When combined, I believe that the ethics of care and feminist political economy can help to abolish the binary notion of masculine/feminine, by emphasizing the fact that all people, regardless of symbolically assigned gender, are located in relations of care.

\subsection{Why the Ethics of Care?}

\footnotetext{
${ }^{22}$ As mentioned in chapter two, Sevenhuijsen also explains that she chooses to still use the term 'feminist' ethics of care to acknowledge the feminist history and genealogy of the ethics of care $(1998,34)$. I agree that besides the fact that I view the ethics of care as feminist in its aims and in its challenges to the masculine-biased political and moral theory, the feminist history of this concept is also a worthy reason to maintain the use of the term feminist ethics of care.
} 
There are several approaches to policy analysis; $\mathrm{Pal}(2010,19)$ notes that each approach is based on a type of reasoning. For instance, legal assessments look at public policy through the prism of law, while logical analyses interrogate the inherent consistency and coherence of a policy (Pal 2010,20). Normative analysis, the type of policy analysis I pursue in the next chapter, evaluates policy in reference to basic values or ethical principles. Such analyses, I believe, are extremely important when considering social policies for two main reasons.

Firstly, policy formation and policy implementation are always implicitly, and sometimes explicitly, based on normative judgments. The definition of social policy alludes to this fact: social policy is "an intervention by governments or other public institutions designed to promote the well-being of its members or intended to rectify social problems" (Hankivsky 2004, 9; quoting Feder Kittay 1998, 569). The identification of problems, the definition of well-being, and the decision that well-being should be promoted, all of which form the basis of policy development, are themselves normative judgments, based on a certain set of ethical standards. Further, once problems are identified and solutions suggested, the implementation of policy has a normative dimension: to implement one policy is to assert that it is better than another, and such assertion must also be based on a particular set of ethical values.

Significantly, this facet of public policy is not always apparent. Policy is often presented as arising from an objective process, based on cold logic and economic reason (which also masquerades as an objective method). Explicitly undertaking normative analyses of policies, I suggest, can help counter this lack of transparency, by bringing the ethical dimension of public policy formation and implementation to the fore. In so doing, 
normative analysis also facilitates a critical examination of the 'hidden' ethical principles that underlie policies that appear to be objective.

Secondly, I also believe that it is important, in and of itself, to carefully consider how policies measure up against our ethical standard(s). Importantly, such consideration also involves the continual interrogation of said ethical standards. Policies inform and shape the lived realities of all our lives; to assess them without consideration for whether or not they adhere to the principles that we believe are important is to create space for misalignment between the way our daily lives are organized and our values. As a corollary to this, however, we must also use the relations and dialogues between policymaking, policies, and ethics to not only assess the policies themselves, but also, to question the standards which are used to evaluate said policies. In this way, we commit to not only investigating the material consequences of particular policies, but also to the ongoing assessment of what our ethical standards are, and whether or not they fit the lived realities of our daily lives. Assessing policies using ethics facilitates the development and critique of both our ethical frameworks and our policies so that they can both evolve to more fully address the problems and needs that shape our material lives.

If we are to pursue policy assessment based on ethics, the next question is why one should use the ethics of care, as opposed to other ethical frameworks, as the ethical standard from which to evaluate social policy. Elsewhere, the ethics of care has been compared and contrasted with several other specific frameworks; Robinson's chapter four in Globalizing Care: Ethics, Feminist Theory, and International Relations (1999) is a particularly useful example of this. I have chosen to approach this question in a slightly different fashion, and in the following sections, I seek to highlight what I see to be the 
main strengths of the ethics of care as compared to other ethical frameworks in a broader sense. By broader sense, I suggest that a few fundamental assumptions and premises underpin some of the more prominent ethical frameworks. These assumptions, as I seek to demonstrate, are problematic. Further, the ethics of care, by starting from different premises, avoids such issues, and, I believe, provides an ethical framework better suited to moral practice.

Following Robinson, I suggest that the central principles and guiding assumptions of rights-based contractual liberalism can be found in both cosmopolitan and communitarian perspectives $(1999,64)$. These principles include notions of objectivity and universality, rights, individualism, and equality (Robinson 1999, 55-65). Although these principles can, and often do, have merit, I argue that ethical framings which privilege these principles without critically assessing them in particular contexts can, in fact, contribute to the (re)production of marginalization and oppression. The ethics of care, in contrast, starts from very different premises, and as I show below, can actually be used as a tool to dismantle systems of oppression, domination, and exploitation. It is for this reason that I believe the ethics of care must be taken seriously as a guide to moral practice for individuals, communities, governments, and nations alike.

\subsubsection{The Myth of a 'True' Ethic}

In Moral Understandings (1998), Urban Walker explores moral epistemology. Epistemology, in general, is the way we theorize knowledge, and in this piece, Urban Walker challenges some common views about "what moral knowledge is like, where to look for it, and how to tell when you've found some" $(1998,4)$. While Urban Walker's 
insights are many, for the purposes of this piece, I wish to explore one in particular: the myth of a true and universal morality and ethics:

Many moral philosophers will say that in their philosophical reflections they are not 'merely' reflecting on their own moral experience (much less mirroring it), but are tapping into moral reality, or the moral realm, or the structure of practical reason, or the nature of the right or the good. But this assumes two things. It assumes that the moral reality, realm, nature, or structure is something accessible and determinate quite apart from anyone's acquired experience of them, and that the moral philosopher can tell when she or he has grasped these things as they really are apart from his or her thoroughly tutored and cultured experience of them. (Urban Walker 1998, 5)

The idea that there is a true, natural, and universal moral realm is, certainly, appealing. It seems to me that it would be a much easier if there was a true ethical and moral framework; the task, then, would simply be to uncover it and follow the guidelines it lays out for us. However, as Urban Walker points out, "wholesale metaphysical and epistemological positions" $(1998,7)$ drastically misconstrue the fact that morality and ethics are practices:

Morality arises and goes on between people; recruiting human capacities for selfawareness and awareness of others' awareness; for feeling and learning to feel particular things in response to what one is aware of; for expressing judgment and feeling in the responses appropriate to them. (Urban Walker 1998, 5)

Morality and ethical framings arise from people, from culture, from our interconnectedness with all things. It is neither universal nor abstract; instead, it is particular and real, and comes from particular and real circumstances.

To illustrate, it is useful to consider epistemology, the theory and study of the development and evolution of knowledge, before turning to moral epistemology. Historically, many types of knowledge have been considered 'truths;' this is perhaps best exemplified by certain theories in the hard sciences. However, several authors have 
demonstrated that knowledge is neither true nor static. In particular, Kuhn's The Structure of Scientific Revolutions (1996) and Fox Keller's Reflections on Gender and Science (1995) have debunked the idea of natural and/or objective truths specifically in the sciences, albeit in two different ways.

Kuhn's concept of paradigmatic shifts demonstrates that knowledge cannot be considered 'true' or absolute. In Kuhn's analysis, the paradigm is a key component of the study of science. It is the framework from which 'normal science' is conducted. In other words, a paradigm provides a meaningful context and framework in which scientists can work towards the solutions of problems in their field. However, these paradigms are not to be considered truths. When anomalies arise, and the current framework cannot sufficiently deal with such anomalies, a paradigm shift can occur, in which the entire framework of knowledge can be challenged and may even change. The fact that such evolutions exist refutes the notion of a singular, natural, and true knowledge, and shows that knowledge and knowledge production is ever-changing. Fox Keller, on the other hand, illustrates how the formation of knowledge is not an objective process. She interrogates the ways in which people's positionalities filter into knowledge production, with a particular emphasis on the masculine bias in science. Her work demonstrates how the social construction of the binary opposition between 'masculine' and 'feminine' has influenced the social construction of science in many ways, particularly in the use of overbearing masculine metaphors. Fox Keller illustrates that knowledge does not spring naturally from 'the abyss,' but rather, it is created, and created through a process which cannot, and must not, be considered objective. Together, these two insights abolish the claim that any knowledge is natural, true and/or objective. 
Like knowledge, morality and ethics can also be questioned and examined on an epistemological level, and indeed, the same findings that Kuhn and Fox Keller illuminate regarding scientific knowledge apply to morals and ethics. Morality can change and is changing, and it does so largely because it is socially constructed. It is, as Urban Walker describes, a product of the exchanges and interactions between peoples, as influenced by the larger landscapes of care in which people reside. Ultimately, morality is a practice, not a theory (and certainly not a universal, true theory at that), and practices are shaped by, and in turn shape, culture, politics, ideology, religion, systems of power and so on.

The implications of this are important. If an ethical framework is premised on the idea of universality and truth, I believe that an understanding of moral epistemology and the ways in which moral knowledge is (re)produced and disseminated severely challenges such a framework. To be more exact, an understanding of moral epistemology, which defines morality and ethics as practices arising from particular contexts, invalidates any ethical frameworks which theorize morality as universal truths. It also makes a compelling case, correctly I believe, that morality and ethical frameworks cannot be discussed without paying attention to the particular contexts in which moral practices and problems arise. This, as has been much discussed, is also one of the fundamental premises of the ethics of care, and one of the characteristics which distinguishes the ethics of care from many of the mainstream ethical frameworks.

In other words, if we take moral epistemology seriously, and I very much believe we should, it becomes clear that any moral and ethical framework which posits universality and abstract truths necessarily violates the understanding that morality and ethics are constructed, subject to change, and that they are practices which arise from 
particular contexts. The ethics of care, however, is not one such framework. Instead, the ethics of care goes hand in hand with this epistemological view. It emphasizes the importance of context, it sees morality and ethics as a practice, and prioritizes the fact that this practice arises through the interconnected webs which we are all a part of. As a final point, the fact that morality and ethics are not theories does not imply that we cannot theorize about such things. As Urban Walker states, it just means that "theories of morality should not be confused with morality, the human social phenomenon the theories are about" $(1998,15)$. It indicates that because morality is not a theory, but certain practices, then "the theory of morality is an attempt to understand these practices" (emphasis in original, Urban Walker 1998, 15). As a part of this attempt to understand these practices, however, it must be underscored that the investigator of these practices is not operating outside the plane of morality and ethics. This is a strength of the ethics of care; care ethicists who truly understand the importance of contextual sensitivity in the ethics of care cannot suggest that they are objective experts of ethics and morality. Instead, as a part of contextual sensitivity, care ethicists must also be placed in the landscapes of care that are in question.

The fact that moral philosophers and ethicists actively participate in the plane of morality is important, as this participation can have far-reaching material consequences (Urban Walker 1998, 4). As Fox Keller demonstrates with regard to science, the particular positionality of the theorist can (often unintentionally) trickle into the ways in which moral and ethical frameworks are described, understood, and discussed. The consequence of this is often the marginalization of some; this is demonstrated in the next section, which discusses the dangers of the rhetoric of equality, individuality, and rights. 


\subsubsection{The Dangers of Equality, Individuality, and the Notion of Rights}

In addition to some ethical frameworks relying on the myth of universality, mainstream ethical frameworks, like liberalism and rights-based ethics, are built on premises of equality, individuality and autonomy, and the notion of rights (Sevenhuijsen 1998, 40). In this section, I argue that while these ideas may, in an abstract sense, have merit, when they are placed within the wider social-political-economic landscape, it becomes evident that a reliance on ethical frameworks which are built on these notions is potentially dangerous, in that they can contribute to the (re)production of marginalization for certain groups of peoples.

The notion of equality is widely used in political arenas today: everyone deserves equal treatment, and equal access to the things that make living possible. However, equality needs to be interrogated more closely. For one, the very notion of equality suggests that equality exists; this is a dubious statement in my mind, but perhaps it can be overcome by arguing that it exists 'for some.' More importantly, however, is that the notion of equality also leads to the conclusion that individuals should be treated as equals under all circumstances, with the expectation that "equality in treatment will lead to equality in results" (Sevenhuijsen 1998, 42). Such logic is dangerous, for "to assume equality among humans leaves out and ignores important dimensions of human existence" (Tronto 1993, 135); in particular, the fact that we oscillate between various states of vulnerability and dependency throughout our lifetimes. Besides ignoring this reality, the idea that equality is a pillar of morality further implies that only those relationships between equals, or those who are deemed equal in some important sense, are the types of relations that morality can regulate: 
Relationships between those who are clearly unequal in power, including large and small states, parents and children, earlier and later generations, have had to be shunted to the bottom of agenda and then dealt with by some sort of promotion of the weaker, so that an appearance of virtual equality is achieved. (Robinson 1999, 61)

When we start with a premise of equality, justice tends to be understood as the act of treating like cases alike (Sevenhuijsen 1998, 40). However, as Sevenhuijsen points out, in order to treat like cases alike, we must reflect on what 'alike' really means. This is where things become complicated. What does it mean to be 'alike'? In order to be considered alike, and thereby treated equally, do the two subjects in question have to be identical? If they are not alike, how do we proceed? How much variation can exist before they are no longer considered alike? And most importantly of all, who determines such criteria?

The very existence of such criteria would suggest a 'norm of equality,' and it is here we see the danger of equality expand. The idea of a norm of equality automatically implies that there are differences between subjects: if there were no differences, then equality as a concept would be redundant (Sevenhuijsen 1998, 43). Assessing whether or not these differences 'matter,' and whether or not they impede from treating like cases alike, requires a standard for making comparisons. Unfortunately (or fortunately, depending where you stand), it also seems likely to me that the criteria on which such assessments are based have historically been, and indeed will continue to be, defined by the norms of the hegemonic order of the day. For example, the norms from which equality is determined would be characterized by the norms of hegemonic masculinity: equals are peoples that are independent, autonomous, rational and objective. Those who do not meet such requirements become 'othered,' as differences are considered deviant from the norm. In this way, a hierarchy also emerges: those who meet the norm of 
equality and, of lesser importance, those who do not. Those who do not reflect the norm, as Robinson's quote above explains, are often 'shunted' to the bottom of the agenda simply because they do not 'measure up' to a certain idea which has been created by the privileged. As Hankivsky notes, the choice of standard will (and does) determine the nature and the final result of equality comparisons $(2004,45)$; if the choice of standard is determined by those with power and privilege, and it seems probable that it is, it is likely that the final result of the equality comparison will only further serve to (re)produce those people's power and privilege, while marginalizing others.

In similar fashion, the notions of autonomy and individuality also manifest in material ways, to the benefit of some and the detriment of others. I have discussed the dangers of the autonomous individual as the prototype of moral subjectivity in the previous section, and for that reason I shall be brief here. However, I must reassert that the idea that the moral subject is individual and autonomous not only excludes those who do not effectively mask ${ }^{23}$ their dependencies from the moral realm, but because it is used to inform government policy, also risks alienating those who do not meet this imaginary standard. Sevenhuijsen writes:

[...] I have always found the objective of government policy of ensuring that every citizen should in principle be able to look after themselves... to be paradoxical. This norm of self-sufficiency and the related view of human nature assumes each citizen to be a detached individual, whose aim is autonomous behavior, who needs nobody and recognizes dependency and vulnerability only in others. It means that care figures in politics as a handicap, as a burden or as a 'necessary evil'... This image expresses a one-sided and, in a certain sense, harmful subject ideal because it encourages citizens also, in the exercise of

\footnotetext{
${ }^{23}$ I use the term 'mask' because I reject the idea that there exists anyone who is fully independent; I believe that some people are simply better at hiding their dependencies, particularly when they are in privileged positions and/or have greater access to resources.
} 
citizenship, to look for needs and problems in others, rather than in themselves. $(1998,28)$

If independence and autonomy are the ideal standard which people aspire to, then government policy will tend to be built around allowing this to happen. Of course, I am not claiming that such policy is entirely negative, nor am I arguing against government support systems which encourage individuals and families to live life in healthy and fulfilling ways on their own terms. However, for a government to focus on such an objective misses a fundamental fact of the human existence. We are all connected, and at times, dependent on others. This should not be a failing. When policy is built around the goal of ensuring that 'every citizen should in principle be able to look after themselves,' then those who are experiencing moments of dependency are seen as lacking, and viewed as a burden on the system. In other words, the ways in which government policy are aimed at maintaining independence and autonomy can often mark individuals with visible dependencies as societal pariahs.

The notion of rights falls subject to similar issues. For one, rights are abstractions, determined once again by the norm. Thus, the same weaknesses arise: what is a right and why? Is this a right for everyone? Who determines what rights are? I suspect that such questions are answered more often than not by those who are privileged and in power. Because rights are constructed, then, they also cannot be thought of as something people 'possess.' Instead, rights should be understood as a competence granted to individuals and families by the state (Sevenhuijsen 1998, 95), based upon the hegemonic ideologies of a particular time and culture. Rights also tend to start from the (incorrect) pretence that 
all people are already equal (Robinson 1999, 64). I have already demonstrated the fallacy of such pretence, and illustrated the danger of assuming such pretence to be true.

Additionally, there is a last point about rights that must be addressed. Rightsbased ethics assume that individuals can always claim their rights. More specifically, rights-based ethics subscribe to the myth of the autonomous individual discussed previously, and clearly, such a person can always access their rights, or at the very least, actively seek out and claim them. This, as Robinson explains, completely ignores the "oppressive and exploitative social and economic structures and cultural norms" (1999, 64) that prevent (certain) people from claiming their rights. By ignoring such structural barriers, this idea also further marginalizes those who face these barriers. Once again, it is clear that the ways in which these premises are formulated are not simply ideological matters to be debated amongst theorists. They have far-reaching consequences as they inform the governance of nations, government policies, and indeed, the lives of all of us.

To conclude, I believe that the issues relating to ethics based on equality, individualism and autonomy, and notions of rights, effectively demonstrate why I have chosen not to rely on ethical frameworks such as liberalism, rights-based contractarianism, cosmopolitanism, communitarianism, and indeed any others which depend on such concepts. The ethics of care, I contend, overcomes the limitations of these ethical frameworks. The ethics of care does not insist on universal truths or abstract notions of morality. It views morality as a practice, which is as unique and varied as the particular relations and landscapes from which moral issues arise. It does not idealize individuals as independent autonomous beings; it seeks to uncover the ways in which our dependencies and relations allow (or alternatively hinder) our ability to (re)produce 
flourishing lives. It focuses on context, and combined with a feminist political economy methodology, pays particular attention to the ways in which power asymmetries vary and shift across landscapes of care. It suggests that by understanding such power asymmetries, we will be better equipped to deal with moral dilemmas. Finally, I believe that by focusing on power dynamics, the ethics of care also provides space to interrogate critically existing systems of exploitation, oppression and domination. By uncovering how these systems impede our abilities to care, the ethics of care can illuminate the material consequences of these systems, bringing them to the forefront of our political discussions, and demonstrating how they are detrimental to us all. 


\section{Chapter: Assessment of the Newfoundland Paid Family Caregiver Program from an Ethics of Care Perspective}

In this chapter, I assess the Newfoundland Paid Family Caregiver program using a critical ethics of care lens. In the previous chapter, I supplied a critique of some of the fundamental premises of traditional ethical frameworks, and argued that the ethics of care overcomes these limitations and should thus be taken seriously as an ethical framework. I also argued that this is particularly true when examining social policy. Newfoundland and Labrador, like the rest of Canada, has been known for its orientation toward welfare provision and its development of social policies and programs (Hankivsky 2004, 30). ${ }^{24}$ Despite the prevalence of social policies in Canada, normative analyses of such policies are rarely undertaken $(\mathrm{Pal} 2010,19)$. In the analysis below, I hope to add to the growing literature that demonstrates the importance of such analyses, particularly when a critical ethics of care is used as the methodological lens from which to approach such investigations (cf. Hankivsky 2004; Mahon and Robinson 2011).

The aim of this chapter is thus twofold. First, I demonstrate the usefulness of the ethics of care as a methodological tool to examine social policy through a case study of the Newfoundland Paid Family Caregiver Program. Simultaneously, I critically examine the Newfoundland Paid Family Caregiver Program, and offer insights into the ways in which this social program is (re)structuring caring relations in this province.

\footnotetext{
${ }^{24}$ Arguably, such an orientation has been shifting in more recent years. However, given that many services, particularly the provision of health care and basic education, are publically provided, I contend that this view still applies.
} 


\subsection{The Case Study: The Newfoundland Paid Family Caregiver Program}

The province of Newfoundland and Labrador, like the rest of Canada, is experiencing a variety of changes which are impacting the ways in which long-term care is provided for the elderly population. These changes can largely be attributed to shifts in demographic patterns, out-province migration, the decay of the traditional male breadwinner model and ongoing cuts in social services (Government of Newfoundland 2012, 6-7). Together, these shifts have contributed to the emergence of critical gaps in the provision of long-term care for the elderly population, particularly in rural areas. In response to this problem, the provincial Conservative Government is in the process of initiating a program whereby eligible home support clients in the province will have the option of paying family members for some personal care and behavioural support services. Having included this program in its 2011 Blue Book (PC Party of Newfoundland and Labrador 2011, 63), the pilot project for this program began on March 24, 2014 (Sullivan 2013). It is notable that the roll out for this program was delayed several times. Given that 2015 is a provincial election year, however, the Conservative Government experienced extreme pressure to initiate the program in 2014, and fulfill the commitments made in the Blue Book. Although this program targets both senior citizens and adults with disabilities, this thesis focuses on the senior citizen population only. ${ }^{25}$

As the project is in the early stages, the overall scope and financial budget for the program is unknown. However, the pilot project has a budget of $\$ 8.2$ million, and at

\footnotetext{
${ }^{25}$ As previously mentioned, the vast literature on disability and care often addresses issues that do not frequently arise in elder care (for instance, disability literature often highlights the oppressive history of care; cf. Kröger 2009; Williams 2001), and thus I have chosen to narrow my focus in this way for pragmatic reasons.
} 
capacity, it will include 250 individuals, from the four health regions of Newfoundland and Labrador (PC Party of Newfoundland and Labrador 2014). These regions are divided geographically into the Eastern, Western, Central, and Labrador/Grenfell Regions (Government of Newfoundland 2014b). Of the 250 participants, 170 spots are designated for senior citizens and the remaining 80 spots are designated for adults with disabilities (Caregivers Out of Isolation Newfoundland 2014). All 250 participants ${ }^{26}$ must be new to the home support system, and an evaluation is required to determine eligibility (PC Party of Newfoundland and Labrador 2014). The criteria to determine eligibility are the same as those used to determine if an individual is eligible for the home support system already in place.

The logic behind the program is to pay family members a wage to compensate and support their care work. Notably, while the program is "broaden[ing] the definition of family members who can provide care to include parents, children, grandparents, grandchildren, siblings and [other] ${ }^{27}$ relatives [who are] residing in the same home," the program excludes spouses/common law partners from receiving financial compensation for their work. These caregivers are excluded "as there is still the expectation that the natural caregiving roles ${ }^{28}$ provided by these individuals will continue" (Sullivan, 2013). Furthermore, it is unknown if this program offers training and support to these family

\footnotetext{
${ }^{26}$ Note that the participants are the care receivers, rather than the caregivers (who are in fact the individuals that will be financially compensated through this program).

${ }^{27}$ I have added the term 'other' because there is an important distinction here which I believe is lost without the inclusion of this word. If you are a parent, child, grandparent, grandchild, or sibling, you can be the paid family caregiver without living in the same home as the care recipient. If you are a family member of any other relation (i.e. cousin, nephew, niece, etc...) you must be living with the care recipient in order to be the paid family caregiver under this program.

${ }^{28}$ While beyond the breadth of this thesis to explore, the assertion that spouses and partners are natural caregivers is extremely problematic. For a discussion of who has the 'capacity to care' please see Hollway (2006).
} 
caregivers, although it seems unlikely. One document states that currently there are no mechanisms to "objectively measure family capacity to care" (Government of Newfoundland 2014d, 8), and as such, the program strictly relies on the caregivers' personal assessment of how much/what kind of care they can provide.

The 'selling point' of this program is that it increases choice for care receivers. Minister of Health and Community Services Susan Sullivan writes, "Our design objective with the Paid Family Caregiving Option is to increase a client's flexibility and choice by making it easier for adults, who require and are assessed as eligible for home support, to hire a family member" (2013). The idea that this program increases choice needs unpacking, however, given the urban/rural divide in how current long-term care services are organized. For instance, out of the 23 publicly funded long-term care homes in Newfoundland and Labrador, 8 are located in the two urban centres, St. John's and Corner Brook (Government of Newfoundland 2014a). Another example which demonstrates a 'double standard' between long-term care services in rural versus urban areas can be found in the Eastern Health region. While there is no official policy at this time, in the Eastern Health region patients in rural hospitals must accept the first available suitable bed within a 100 kilometer radius of their home area, whereas patients in St. John's are not expected to leave the city even if there is a suitable bed available outside of the city limits (J. Dalton, pers. comm.). While this is, as stated, more of a practice than a policy, rural individuals are more likely to be separated from their family and community, and have less choice in terms of their placement, than their urban counterparts. This suggests that while not acknowledged by the program itself, rural participants may in fact have a different set of criteria to consider when making the 
'choice' of whether or not to participate in this program, as compared to those in urban areas. Further evidence of this rural/urban divide is evident when financial budgets are compared. For instance, while the pilot project for the Paid Family Caregiver Program is operating on a budget of $\$ 8.2$ million to assist 250 individuals (mostly from rural areas) (DOHCS 2014), the government has recently invested $\$ 150$ million in a long-term care facility in St. John's for 375 individuals (Belac 2014). Again, this illustrates the unequal distribution of long-term care resources between the rural and urban populations of Newfoundland and Labrador, and given the large geographic spread of the province, this means that unless one is willing to relocate to the urban areas, the options for publically provided long-term care are extremely limited. Because of such issues, the claim that this program increases 'choice' for those in need of long-term care requires further investigation. As of right now, the pilot project of the Newfoundland Paid Family Caregiver has geographically distributed the subsidies under this program as follows:

\begin{tabular}{|c|c|}
\hline Region & Subsidies for Seniors \\
\hline Eastern & 85 \\
\hline Central & 35 \\
\hline Western & 30 \\
\hline Labrador-Grenfell & 20 \\
\hline
\end{tabular}

Table 4.0. Geographic distribution of subsidies. Source: Government of Newfoundland 2014d, 42.

While the Conservative party has advocated for this program, members of both the Liberal and New Democratic parties have voiced concerns related to this initiative. In particular, the Liberal party takes issue with the fact that the program took so long to develop, and that information about the program has been notoriously hard to access (Liberal Party of Newfoundland and Labrador 2014). Since the news release confirming the start date of the project, however, the Liberal party has been surprisingly quiet on the 
matter. The NDP, on the other hand, share this concern but also have a more longstanding worry. With the massive layoffs of Government employees that occurred in the spring of 2013, members of the NDP wonder how the already-stretched staff will be able to train for this new program, and effectively administer and manage it (NDP of Newfoundland and Labrador 2013). While members of the NDP have publically expressed this concern, no direct reply from Conservative politicians has been made at this time. Other groups, including the Caregivers Out of Isolation Group, a network of family caregivers in Newfoundland and Labrador, have also been generally silent in sharing their opinions/assessments of the program. ${ }^{29}$

\subsubsection{Logistics of the Program}

This program retains the principles of the current home support system in Newfoundland and Labrador, but removes the current barriers which prevent family members from being paid for caregiving (Government of Newfoundland 2014d, 6). When an individual is interested in participating in this program, they are assigned a case manager who assesses the individual's eligibility. In order to be eligible for this program, the individual must meet the eligibility requirements for publically funded home support, which are outlined in the Provincial Home Support Program Operational Standards (2005), and which are based on (1) need for service, (2) place of residence, and (3) financial eligibility. The Newfoundland Paid Family Caregiver Program particularly

\footnotetext{
${ }^{29}$ To my knowledge, only two newspaper articles provide an opinion of the program by a caregiver. One article, by CBC (2013), interviews a mother with a severely disabled adult son. The mother's main point of contention with the program is that the pilot project excludes people who already receive home care (as mentioned above, only new individuals to the home care system in Newfoundland and Labrador can apply for this program). The second article also interviews a mother who cares for her disabled son, and who is concerned that there needs to be more respite available to family caregivers (Crocker 2013). Again, to my knowledge, no article engages with care receivers.
} 
emphasizes that the individual must demonstrate that they face an unmet personal care/behavioral support ${ }^{30}$ need. The individual must be 'a new client,' which is defined as an individual who has not previously received service or an individual who has been with no service for a period of one year or more from the Adult Home Support Programs [which are publically provided]. (Government of Newfoundland 2014d, 14)

Once an individual is deemed eligible for the program, a 'plan of care' is developed based on the assessment of the individual's needs. This assessment is conducted by the individual's case manager. While each plan will be unique to the individual, there are maximum thresholds for the eligible services. These are summarized in Table 4.1:

\begin{tabular}{|c|c|c|c|}
\hline \multicolumn{4}{|c|}{ Summary of Maximum Hours of Care Supported by the Newfoundland Paid Family } \\
Caregiver Program
\end{tabular}

Table 4.1. Source: Government of Newfoundland 2014d, 17.

The total monthly home support ceiling for seniors is $\$ 2810.00$ (Government of Newfoundland 2014e). Thus, respite care funds are provided if and only if the cost of the care provided by the family caregiver does not exceed the total monthly home support ceiling of $\$ 2810.00$. Respite funding is only available to those clients who are assessed as requiring 24-hour care and supervision and respite cannot be provided by other family

\footnotetext{
${ }^{30}$ Behavioural support is defined as "the provision of supervision to clients that have assessed behavioural support needs. It includes services to support clients where there is risk of harm to self or others and to support independence in the home and community" (Government of Newfoundland 2014b).
} 
members (Government of Newfoundland 2014d, 15). This ensures that under the Newfoundland Paid Family Caregiver Program, only one family care provider is compensated for their care work.

The 'Homemaking' category is further divided into the categories below:

\begin{tabular}{|l|l|}
\hline \multirow{5}{*}{ Household Management/ Homemaking } & $\begin{array}{l}\text { Up to 1 hour/day for meal preparation and } \\
2 \text { hours/week for homemaking when the } \\
\text { caregiver does not reside with the client }\end{array}$ \\
\cline { 2 - 2 } $\begin{array}{l}\text { Up to 2 hours/week of homemaking when } \\
\text { caregiver resides with client and the } \\
\text { client's care needs results in additional } \\
\text { homemaking requirements }\end{array}$ \\
\hline
\end{tabular}

Table 4.2. Breakdown of household management/homemaking. Source: adapted from Government of Newfoundland 2014d, 15.

The distinction here lies in whether or not the family caregiver lives with the care receiver. If the caregiver does not live with the care recipient, they can be compensated for meal preparation up to a maximum of 1 hour/day. If they do reside in the same household as the care receiver, however, they cannot be compensated for any meal preparation at all. While the reasoning behind this distinction is not explicitly stated, I suspect that the logic behind this is that if the caregiver lives in the same house as the care recipient, they are cooking meals for themselves, and as such, there is (apparently) no 'added work' to cook meals for the care receiver. Therefore, while there is a 'Homemaking' category under this program, it is clear that "payment of family members [is] primarily for personal care/behavioral support" (Government of Newfoundland 2014d, 12), as very little compensation is allocated for household management tasks and work. The distinction between maximum payments based on whether or not the caregiver resides with the care recipient can be summarized as follows: 


\begin{tabular}{|c|c|c|c|c|c|}
\hline \multicolumn{6}{|c|}{$\begin{array}{c}\text { Detailed Breakdown of Maximum Hours of Care Supported by the Newfoundland Paid } \\
\text { Family Caregiver Program }\end{array}$} \\
\hline & & $\begin{array}{c}\text { Personal } \\
\text { Care/Behavioral } \\
\text { Support } \\
\end{array}$ & $\begin{array}{c}\text { Household } \\
\text { Management/ } \\
\text { Homemaking }\end{array}$ & $\begin{array}{c}\text { Maximum } \\
\text { Total per } \\
\text { Week } \\
\end{array}$ & Respite \\
\hline \multirow[b]{2}{*}{ Seniors } & $\begin{array}{l}\text { Caregiver } \\
\text { Residing } \\
\text { with Care } \\
\text { Recipient }\end{array}$ & 4 hours/day & 2 hours/week & 30 hours/week & \multirow{2}{*}{$\begin{array}{l}\text { Up to the } \\
\text { balance } \\
\text { within the } \\
\text { financial } \\
\text { ceiling by an } \\
\text { agency } \\
\text { (preferred) or } \\
\text { a non-family } \\
\text { member }\end{array}$} \\
\hline & $\begin{array}{c}\text { Caregiver } \\
\text { not } \\
\text { Residing } \\
\text { with Care } \\
\text { Recipient }\end{array}$ & 4 hours/day & 9 hours/week & 37 hours/week & \\
\hline
\end{tabular}

Table 4.3. Source: adapted from Government of Newfoundland 2014d.

Once a plan of care has been established, the client must register with the Canada Revenue Agency (CRA), administer a payroll to the caregiver, maintain employment records, and make the appropriate CRA remittances (Government of Newfoundland 2014c, 4). Recall that the client is the care recipient. The client does have the option of relying on a book keeper, or other such individual, to conduct these tasks on their behalf; ${ }^{31}$ this person, whether it be the client themselves or a third party, is known as the 'Funding Administrator' (Government of Newfoundland 2014d, 31). The funding administrator receives the funding from the Government of Newfoundland and Labrador on a semi-monthly basis, and becomes the employer of the caregiver. While documentation must be kept by the funding administrator, clients are not required at this time to submit timesheets for service hours (Government of Newfoundland 2014d, 38). The funding administrator is required to keep a 'Record of Support Journal;' this is to be

${ }^{31}$ This person cannot be the paid family caregiver. 
reviewed with the case manager on quarterly bases. Audits are also possible under this program (Government of Newfoundland 2014d, 33; 36).

Finally, if the Paid Family Caregiver option is chosen, it cannot be combined with any other public funding/support services. The participant can, however, choose to remove themselves from the program, and will then restart the assessment process to see if they are eligible for other public care services.

\subsection{Assessment}

A normative assessment of the Newfoundland Paid Family Caregiver Program using a critical ethics of care lens illuminates that this program is fraught with tensions in terms of how this program is restructuring care work, and how this restructuring will affect those who give and receive care. By drawing upon the concepts described in chapter three, specifically, contextual sensitivity and the moral subject, attentiveness, responsibility, competency, and responsiveness, I probe these tensions, and argue that these tensions are useful starting points from which to understand the wider implications of this program.

\subsubsection{Contextual Sensitivity and the Moral Subject}

First and foremost, several points of importance arise when one considers the Newfoundland Paid Family Caregiver Program in terms of contextual sensitivity and the moral subject. While each individual who wishes to be considered for this program is assigned a case manager, who will presumably pay attention to the unique circumstances in which this individual's needs arise, I suggest that because of the ways in which the assessment of needs is conducted, this type of contextual sensitivity can only provide a partial picture of the individual's caring landscape. According to the Provincial Home 
Support Program Operational Standards (2005), the three criteria which are used to assess an individual's eligibility for long-term care support are based on (1) need for service, (2) place of residence, and (3) financial eligibility. The need for service criterion uses an assessment instrument to determine functional need and to aid in the development of a service plan; the place of residence criteria is met if the individual lives in a 'self contained residential unit,' such as a house, condominium, or apartment; and lastly, financial eligibility is evaluated based on the individual's liquid assets, income and living expenses (Government of Newfoundland 2005, 8). Clearly, the evaluation of these criteria requires that a certain amount of attention be paid to the context in which the need arises. At the same time, however, there are many facets which such a narrow assessment cannot possibly include.

For instance, while the need is assessed, no attention is given to how that need arose in the first place. Because of this, I contend that it will be difficult to use these assessments to implement preventative health care services. To be sure, this is not a stated objective of this program; however, I believe that that, in and of itself, is telling. The long-term care system in Newfoundland and Labrador is operating on a reactionary level; the Newfoundland Paid Family Caregiver Program is one example of such reactionary policy. This is not to suggest that reactionary policies are somehow 'bad;' indeed, they are an important component of health care. However, I believe that a holistic approach to long-term care must involve both preventative and reactionary policies. The ethics of care highlights the fact that we are all embedded in and dependent on relations of care throughout all of our lives. The approach of reactionary policy ignores this reality. Instead, it frames care as something that arises when the individual somehow does not 
meet the necessary conditions of self-sufficiency; it suggests that the government reacts to the failure of individuals. In contrast, I believe that social policy which takes care seriously would involve continual care policies, in which the creation, maintenance, and reparation of care relations is the utmost priority. A policy that is only focused on 'fixing' problems in care relations after they have reached a certain point cannot, in my opinion, be said to fully understand the importance of care in all of our lives.

Similarly, the use of the residential and financial criteria, while seeking to understand the individual's position in terms of a simplified status (i.e. homeowner, asset owner, etc.), misses an opportunity to interrogate the larger context in which that individual lives. I believe that statuses, like home owning, are important signalers of the individual's position within larger social, political, and economic systems. If the Newfoundland Paid Family Caregiver Program were to consider seriously issues of context, these criteria would not simply involve the allocation of statuses to these individual's case files. Rather, these statuses would be used to place the individual in the larger social framework. The insights gained from placing the unique realities of individual's lives into a larger picture could again be used to better understand the larger context in which caring needs arise. This information could inform preventative policy, help us allocate resources to those who need it most, and most importantly, allow us to identify and work to rectify structural issues which negatively affect people's ability to live healthy and flourishing lives.

These issues, I believe, stem from the fact that clearly, this program relies on a view of the (moral) subject as the individualistic and independent man, as opposed to the moral subject of the ethics of care, who is firmly implanted in wide and complex webs of 
relations. The assessment which determines if the person is eligible for long-term care support depends on a view of people as atomistic individuals. The individual is not considered in relation to the social relations of production or other relations of power, or even, it would seem, in terms of the immediate relations of family and friends. Instead, they are assigned statuses without any consideration for how these individuals came to be in such positions and without any exploration of how the caring need reached its current form. They are deemed 'no longer self-sufficient,' and as such, defective and in need of care, as opposed to the unflawed people who can 'care for themselves.'

If, on the other hand, the moral subject of the ethics of care was the subject in this program, I believe the result would be very different. Instead of being assessed as no longer able to look after oneself, the questions involved in the assessment would seek to uncover how the caring needs of the individual have changed (for caring needs themselves exist for all of us at all times, they just take varying forms). Special attention would be paid to understanding how the individual's positionality has contributed to this change, and to the larger obstacles which may be negatively impacting this person's caring relations. The program would not be built around assessing the individual's 'failure' to be self-sufficient, but rather, it would aim to understand the failure of the web of caring relations, ranging from the micro-level of family and friends to the macro-level of social policy, to adequately adapt to changes in the nature of care that occurs throughout the life course.

\subsubsection{Attentiveness}

The Newfoundland Paid Family Caregiver Program facilitates attentiveness in that the elderly in need of long-term care are provided with monetary resources which are 
used to hire a caregiver. In other words, the program makes attentiveness possible by providing financial support for the care relation, in which the needs of the care receiver can be addressed. However, it is apparent that many of the issues that are identified when contextual sensitivity is considered are once again evident when attentiveness is contemplated; for this reason, I will be brief here. Attentiveness requires the careful consideration of the other person's unique and particular reality. As I argued previously, the Newfoundland Paid Family Caregiver Program does not include a platform or mechanisms to facilitate a thorough investigation of the context in which the need for long-term care arises. It focuses on a surface understanding of an individual's needs, without consideration for the complex webs of relations that contribute to the shaping of said needs. It also does not (necessarily) consider whether or not caregivers will be attentive to the needs of the care receiver, or the particular context in which those needs arise. There is no formal and consistent monitoring basis, and no training is expected to be provided to the caregivers; while individual caregivers may have the skill set required to attentively provide good care, others may not. Thus, while it can be suggested that the Newfoundland Paid Family Caregiver Program facilitates attentiveness, in that it provides a monetary incentive to (re)produce a care relation between the family caregiver and the elderly care receiver, it does little to promote true attentiveness, which requires ongoing work, and in which the caring landscape is constantly (re)assessed and the needs of all people in the care relation are continually interrogated.

\subsubsection{Responsibility}

The Newfoundland Paid Family Caregiver Program is interesting to consider when the question of responsibility is posed. In the ethics of care, responsibility does not 
simply imply that someone (or some group) must take responsibility for care. Rather, from an ethics of care perspective, responsibilities are understood to be already-always woven into our lives. The pertinent question is how are responsibilities distributed, and how do the existing political-economic systems, cultural practices, and power dynamics affect this distribution?

The Newfoundland Paid Family Caregiver Program is undoubtedly redistributing responsibilities for care in the province. This government initiative, rather than providing professional or public caregiving services, places the burden of caregiving and receiving back on the individual or nuclear family unit. While it is true that the government is taking financial responsibility for caregiving and receiving, it also simultaneously allocates responsibility to both the family caregiver, as they must do the care work required to address the caring need, and the care receiver, who must become the employer of the caregiver and accept the responsibilities that come along with that designation. This particular redistribution of the responsibility for caring activities can be considered in terms of Esping-Anderson's theory of the welfare state $(1990 ; 1999)$.

Esping-Anderson defines the welfare state as the state's responsibility for securing some basic modicum of welfare for its citizens and for granting social rights (1990, 18-21). However, he further argues that the definition must go beyond this:

The welfare state cannot be understood just in terms of rights it grants. We must also take into account how state activities are interlocked with the market's and the family's role in social provision. $(1990,21)$

Attempting to expand the definition of the welfare state in this way, Esping-Anderson developed three 'worlds of welfare capitalism:' liberal regimes, social democratic regimes, and conservative regimes $(1985 ; 1990)$. The two key variables used to identify 
these three regimes are, as Mahon summarizes, "the degree to which policies foster citizen solidarity versus segmented and stratified systems, and commodification versus decommodification (the degree to which the system of social entitlements mitigates the cash nexus)" (2002, 6). Later, Esping-Anderson also added a third variable: defamilialization, "the degree to which households' welfare and caring responsibilities are relaxed - either via welfare state provision, or via market provision" $(1999,51)$. This last characteristic is of particular relevance for the Newfoundland Paid Family Caregiver program, which is, in fact, re-enforcing the family's care responsibilities.

The first of the three regimes is the liberal regime, which is open to defamilialization, but generally by substituting the family for market initiatives (1990, 26). In this way, public support is largely provided through tax benefits or credits/deductions and transfers. The second regime, social democratic, is characterized by universalism and decommodification of social rights, and the promotion of "an equality of the highest standards, not an equality of minimal needs" $(1990,27)$. This regime is supportive of defamilialization, and offers comprehensive protection from market-generated risks. Finally, the conservative welfare regime provides protection of rights but in a way that preserves status; "rights, therefore [are] attached to class and status" $(1990,27)$. Mahon further notes that this regime has been the most resistant to defamilialization $(2002,7)$.

Within this framework, I contend that the Newfoundland Paid Family Caregiver Program falls under the conservative welfare regime. The very eligibility of the elderly to partake in this program is determined by statuses, such as financial classification. If, based on such statuses, one is deemed eligible, then the program does indeed protect 'the 
right' to long-term care for elders, but it relies on families to provide this care. In other words, this program is 'refamilializing' care, by directly redistributing the responsibility for caregiving and care receiving from the state to the family unit.

This redistribution is of particular interest when one considers the added issues that arise when obligation is considered. The responsibility criterion is distinct from obligation. However, I would suggest that depending on the distribution of responsibilities, feelings of obligation become more or less likely. In the case of the Newfoundland Paid Family Caregiver Program, I fear that a consequence of this 'refamilialization' of care may be that family members feel obligated to provide care. If this program is the only probable or most desirable option available to elderly individuals in need of long-term care, families may feel especially pressured to partake in the program, even when their own material and emotional realities do not realistically allow for such added responsibilities. This could have very real consequences in terms of the provision of quality care, and in terms of the well-being of the caregivers, care receivers, and the family unit as a whole.

To be fair, while I suspect it is unlikely, it is possible that the opposite effect occurs. Perhaps the financial compensation involved in this redistribution of caring responsibilities will relieve family caregivers from a sense of obligation, as they may feel that they are doing this work for personal monetary gain. At this point, further research is required to understand how, if at all, this program changes family member's feelings of responsibility and/or obligation to care. The point, however, is that the nuanced distinction between responsibility and obligation, which is emphasized in the ethics of care, provides a fruitful angle from which to approach the question of the material 
consequences of this program's redistribution of caring responsibilities. Will family caregivers feel obligated to provide care, even when they are unable to do so effectively? If this occurs, what will it mean for the quality of the care provided? Further concerns arise when the question of resistance to this program is considered. For example, if caregivers feel obligated to do care work because of this program, will they be more likely to voice concerns about the way this program is (re)structuring care? Or will they, because of a sense of obligation and magnified social norms which suggest that families are natural caregivers, subdue such resistance and endure the arrangement 'out of duty'? I do not have the answers to such questions. However, the value of assessing social policies such as this through an ethics of care lens is that potential pockets of tensions, like this one, are illuminated, and can help direct further research and assessment of the policy in question.

\subsubsection{Competency}

The criterion of competency once more reveals an important aspect of this program. If we are to have a meaningful ethics of care, in which the consequences of the care relation are successful, then competency must be a part of this. However, the Newfoundland Paid Family Caregiver Program does not assess the ability of the family caregivers to provide effective, quality care. It does not assess the context in which caring needs arise, thereby ignoring larger structural obstacles that may negatively impact the caring relation. And finally, it is unclear if the program will provide training to the family caregivers.

This is not to suggest that all of the family caregivers that participate in this program will provide incompetent care, nor do I wish to imply that the family caregivers 
are not well-intentioned. However, while some caregivers might be fully equipped to perform the care work and provide high quality care, others may not. Without some sort of process to ensure that care is consistently and competently provided by caregivers in this program, I believe that this program fails to facilitate competent care.

As a final point on competency, I must also raise the issue of respite care. Under this program, the conditions under which respite care for the caregiver is provided are problematic. Funding for respite care is only available if the total monthly home support ceiling of $\$ 2810.00$ is not exceeded, and if the case manager concludes that the care recipient is in need of care 24 hours a day, seven days a week. However, a contextually sensitive analysis of the care relation would not default to a generalized rule about how much care must take place before the caregiver deserves relief. Instead, a contextually sensitive analysis, as espoused by the ethics of care, would take into consideration the caregivers' other responsibilities, and the resources and support available to the caregiver. The dangers of applying a generalized respite 'rule' are immense when competency is considered. While some people, for example, may be able to provide quality competent care for 10 hours a day before they require relief, others may only be able to provide 2 hours. This is not to say that one individual is a naturally better caregiver than the other; rather, this is to acknowledge that we are located in different positions in different landscapes of care, with different power, access to resources, and privileges. All of these factors inform our caring relations; without a respite system in place to account for such factors, the provision of consistent, competent care, is in question. 


\subsubsection{Responsiveness}

The element of responsiveness is meant to address the power relations inherent in all care relations - both the caregiver and care receiver must have space to respond to the care relation. The Newfoundland Paid Family Caregiver Program, however, does not have a concrete and systemic way to facilitate responsiveness. While it could be argued that the care recipients, who are the formal 'participants' of the program, can voice concerns and provide feedback to their case managers, this does not ensure that responses will be heard at the appropriate level or taken seriously when the program is evaluated at the governmental level. Furthermore, the family caregivers are not considered to be participants in the program at all, despite the fact that the program has very real implications for the ways in which they provide care, and indeed affects the structure of their lives in general. Instead, under this program, the family caregivers are formally employees of the care recipients.

Without a platform for both caregivers and care receivers to respond to the ways in which this program (re)organizes care, several questions arise: Will caregivers and care receivers be able to equally voice their concerns related to this program? Will caregivers and care receivers be more likely to voice concerns about the way this program organizes care because they are kin, or will this fact deter them from doing so? This last question points to an important observation about the power dynamics involved in the caregiver-care receiver relation, specifically under this program. Relations of care, like all relations, involve power dynamics. The Newfoundland Paid Family Caregiver Program involves at least two additional power dynamics, although in particular circumstances, an attentive, contextually sensitive assessment would likely reveal many 
more. For one, the care relation under this program is shaded by the power dynamics involved in family relations. Secondly, to further complicate such dynamics, under this program the family relation takes on the additional characteristic of an employeremployee relation because the care recipient becomes the employer of the family caregiver. Employer-employee relations further involve complex power divisions. Based on the ethics of care, I make the normative judgment that these power dynamics must be unpacked so that we can better understand how these dynamics inform the care relation. To do this properly requires attentive contextual sensitivity and safe spaces for both parties to responds to the care relation. Notably, as I have demonstrated previously, neither of these are built into the Newfoundland Paid Family Caregiver Program, and for this reason, I see this program as failing to expedite responsiveness.

\subsubsection{Monetized and Targeted Care}

In addition to downloading the responsibility for care onto the family unit, this program also involves the monetization of care, as opposed to the commodification of care. This distinction, while nuanced, is significant; Mahon and Robinson explain:

Monetization involves payment of wages and salaries to care workers, but access to care services is structured by the rules of citizenship. In other words, there is a right to publicly financed (and often publicly provided) care. Commodification accompanies monetization when access to (paid) care is made through the market. $(2011,7)$

Monetization means that the care service itself is still funded by the government, and access to the care service is publically provided. Commodification involves the provision of care through the market, and access to the care service is instead dictated strictly by one's ability to pay. 
The Newfoundland Paid Family Caregiver program does not put care services in the market. While care is monetized, and wages and salaries are paid to the family caregivers, care is not commodified under this program. The program is not for-profit, and it does not exist in the market realm. However, even though this program publically provides care, it is important to note that the provision of care under this program is not universal; rather, this program is a targeted social program. Mkandawire explains:

Under universalism, the entire population is the beneficiary of social benefits as a basic right, while under targeting, eligibility to social benefits involves some kind of means-testing to determine the 'truly deserving.' $(2005,1)$.

The Newfoundland Paid Family Caregiver program is certainly a targeted program; there is a specific process of 'means-testing' which determine if one is eligible to participate in this program or not. The eligibility criteria for this program are first, need, second, residential status, and third, financial status. While these are listed in an order, this is not to signify importance or a weighting system; rather, all three of these criteria must be met in order to be eligible for this program. What is interesting about this system, in my opinion, is that two out of these three criteria are, in fact, indicators of ability to pay. Financial status is clearly an indicator of 'ability to pay;' it is an assessment of one's ability to participate in the market based on one's liquid assets, income, and expenses. Residential status, I suggest, is also an indicator of ability to pay or at the very least, it is strongly related to ability to pay. Residential status indicates if you are a homeowner, if you can afford rent, if you live in a condominium. However, behind these basic labels lies the determining fact: can you afford to pay for these things? Clearly, one's ability to pay is prominently factored into the determination of one's eligibility for this care service. 
While I think that there can be merit in targeting resources to those most in need, the fact that this is a targeted program, and that the eligibility criteria is heavily based on financial status, has some key issues. For one, as Mkandawire notes, "targeting itself almost by definition leads to segmentation and differentiation" $(2005,7)$. It involves the identification of 'the needy,' who require public assistance, while those who are well-off and accordingly 'independent' can (supposedly) access services through the market (Mkandawire 2005, 7). This dual system, however, only serves to further enhance class divisions and stigmatize participants; the elderly care receivers in the program may come to be seen as 'drains' on public resources, given that they are deemed unable to financially 'care for themselves.' Furthermore, when the eligibility criteria for such targeted programs are strongly linked to financial status, the targeting of said programs is not simply aimed at 'those in need;' rather, it is aimed at 'the needy poor.' It appears to me, then, that under this program, financial need may take priority over the actual caring need (which the program is apparently designed to address).

Specifically, targeting necessarily involves a mechanism that discriminates between those who are 'needy' and those who are not; this, however, runs the risk of committing either type I errors, whereby someone deserving of the service is denied access, or type II errors, in which an 'undeserving' person is able to access the program/service (Mkandawire 2005, 9). Under the Newfoundland Paid Family Caregiver Program, I believe that these errors are even more likely to occur. This program is supposed to address a caring need. However, the eligibility criteria are heavily linked to financial status. It seems to me that the assumption is made that caring needs and lowincome are somehow necessarily correlated; what, then, of the people who may not be 
'financially' needy, but who have unmet caring needs? When one considers the geography of Newfoundland and Labrador, I think this is an especially pertinent issue. The lack of both public and market-based care services in the rural areas of the province means that, in some instances, financial resources are irrelevant when attempting to meet caring needs. For example, a person can be quite 'well-off,' but because there are literally no services in their area and because they have no family willing to care for them (whether for pay or for not), they can still have unmet caring needs.

My point is that besides the stigmatization that often occurs when targeting is used to grant access to services, the fact that financial status is such a significant factor in determining eligibility is problematic: if long-term care is to be publically provided on a selective basis, then the unmet caring needs of particular individuals should be the most important criteria in determining who 'deserves' access to this resource. If this is not the case, then the likelihood of type I and type II errors increase. Thus, while financial resources play a role in shaping the unmet caring needs, as a criteria of eligibility, financial status must be examined in reference to the larger context of the individual's life, in relation to the support systems available to said person, and in dialogue with the unique barriers faced by the person in their particular landscapes of care.

\subsubsection{Reflections on 'Choice'}

The participants in the Newfoundland Paid Family Caregiver Program are the elderly care receivers, and as previously noted, the logic behind this program is to provide these participants with 'increased choice' in terms of how they wish to have their caring needs met. Such policy framing and logic reflects a neoliberal ideology. 
Larner provides a detailed definition of neoliberalism, based on three main categories: neoliberalism as policy, neoliberalism as ideology, and neoliberalism as governmentality (2000). Neoliberalism as policy is marked by a shift from a strong, welfare state, to a political agenda focused on enhancing economic efficiency, competitiveness, and facilitating the market provision of goods and services (Larner 2000, 6-7). Neoliberal ideology is distinguished by the moral subject as individual, freedom of choice, market security, laissez faire, and minimal government (Larner 2000, 7). Neoliberalism as governmentality is based upon a useful distinction between government and governance:

[...] While neoliberalism may mean less government, it does not follow that there is less governance. While on one hand neoliberalism problematizes the state and is concerned to specify its limits through the invocation of individual choice, on the other hand it involves forms of governance that encourage both institutions and individuals to conform to the norms of the market. (Larner 2000, 12)

I believe that the Newfoundland Paid Family Caregiver Program's focus on choice reflects neoliberalism as ideology in particular (although choice is a prominent fixture in neoliberalism as policy and neoliberalism as governance as well). Participants in this program are framed as "active agent[s] both able and obliged to exercise autonomous choice" (Larner 2000,13), and the central goal of this program, according to Government press releases (cf. Sullivan 2013) is to provide 'more choice' to those in need of long-term care. This framing, I believe, extends neoliberal ideology by perpetuating and prioritizing 'choice' as a principal value in our lives. ${ }^{32}$ The issue of

\footnotetext{
${ }^{32}$ Larner, drawing upon the work of Stuart Hall (1988), makes the important point that the dissemination of neoliberal ideology is not simply a 'top-down' phenomenon. Rather, the (re)production and extension of neoliberal ideology comes from varying scales across the social-political-economic landscape; part of the reason why ideologies like neoliberalism are so successful as hegemonic ideologies is that "its discourses
} 
whether or not the caring need is met is secondary to the construction of alternative 'choices.'

To be clear, I am not implying that care receivers should not be able to express their preferences in the care relation; as I have argued throughout this thesis, responsiveness, and space for everyone involved in the care relation to voice their concerns, reflections, and thoughts, is a crucial component of good care. Instead, I am suggesting that under neoliberalism, choice is reified to imply a basic act of free will. A critical ethics of care challenges such a simplistic understanding of choice: "from the standpoint of a relational ontology, free choice means more than simply an individual exercise of will; it requires a complicated interaction with the structural conditions of life" (Tronto 2013, 89).

In other words, people who are oppressed, exploited, or dominated, do not face the same set of choices as people who are privileged. Our positionalities, the material world in which we live, and larger social-political-economic structures determine the set of realistic alternatives that we face when making decisions, and influence and restrict our ability to choose freely from such alternatives. A program which claims to increase choice without interrogating the context in which the caring need arises, and without consideration for the particular locations of real people in need of care in the real material world, cannot be said to provide alternative, meaningful options for long-term care

about the world [make] sense to people in a range of different social positions" (Larner 2000, 9). Therefore, I think it is necessary to note that while the government espouses 'choice' as the basis for this program, it is likely that citizens of Newfoundland and Labrador have also adopted such discourse, and asked for 'more choice' in terms of policies related to long-term care. To demonstrate, Minister of Health Susan Sullivan notes that in the process of establishing this program, they "heard clearly from Newfoundlanders and Labradoreans" who "want the option of the choice" to pay family members for care (Crocker 2013). 
arrangements. The prioritization of choice under neoliberalism, and this long-term care policy in particular, is brought into question under a critical ethics of care lens, which unveils the way the neoliberal conceptualization of 'choice' is removed from the complex, material reality, in which people make decisions and live their lives.

\subsubsection{Care as a Social and Collective Responsibility}

The Newfoundland Paid Family Caregiver program not only fails to challenge the characterization of care as a family/private issue, but indeed, it reinforces such an understanding of care. For one, it literally (re)shapes the caring relations of the participants of this program by defining them based on familial relations. Care is pushed (back) into the household; it is not provided socially under this program, nor is caregiving and care receiving treated as a public issue. Secondly, the prioritization of 'choice' under neoliberalism has further consequences in terms of the way we understand our lives and our care needs and relations more generally. Tronto writes:

From the standpoint of a neoliberal society, then, human life is viewed as the sum of an individual's own 'choices,' for which he or she will be responsible. Care thus becomes entirely a personal and private matter; individuals make 'choices' about care for themselves and for those around them. $(2013,40)$

Thus, not only is care literally sequestered in the private sphere under the Newfoundland Paid Family Caregiver Program, but the ideology behind this program, with its emphasis on choice, further pigeonholes care as a private concern, and a private concern alone.

Such an understanding of care is inadequate. As the ethics of care reminds us, we are all embedded in multidimensional webs of relations; these range from individual, personal relations, to larger economic systems, political institutions, and social and cultural structures. Care relations are also embedded in such webs. To assume that care 
occurs solely in the private sphere is to ignore the interconnectedness of all peoples, institutions, states, and systems, and to ignore the ways in which these connections shape caregiving and care receiving. Care is a social and collective responsibility because care is directly shaped by the social and collective systems of our lives.

When care is understood in this way, it makes little sense to relegate care to the 'private sphere.' Care transcends all boundaries, whether real or imaginary, as it is shaped, and shapes, our relations across all contexts and landscapes. To say that care is a collective concern is not to say that care needs cannot (or should not) be met 'privately;' rather, it is to assert that the question of how care needs are met is one which demands social and public consideration and deliberation. Indeed, as Tronto notes, if we could all automatically meet our own needs, there would be no need for politics and political institution, which involve "meeting needs in a way that permits the pursuit of other goals as well, and [...] making decisions about who does what for whom" $(2013,49)$. The fact that we all need care, and are dependent and interdependent on others for this care, is a fundamental reason why political processes exist.

From a critical ethics of care perspective, then, the fact that the Newfoundland Paid Family Caregiver Program fails to challenge the myth that care is a private concern (and indeed, actually reinforces said myth), is problematic. The state itself plays a significant role in the ways in which activities of care are shaped, as exemplified by the way in which this program is (re)organizing care relations in the province. The framing of this program obscures this fact by emphasizing individual choice and family-based relations of care; this must be uncovered, and the role of the state in structuring caregiving and care receiving must be acknowledged and brought to the fore of the public 
discussion. This is not to suggest that the state should become the provider of all care services. Rather, this is to eradicate the perceived divide between the public and private by locating activities of care across all scales and spaces, so that collectively, we can begin to focus on the creation of healthy, flourishing relations of care for all people.

\subsection{A Final Thought: Imagining Alternative Policies}

What might a policy, built on the tenets of a critical ethics of care, look like? This is a salient question; while the analysis above illustrates how the ethics of care can be used to disassemble, uncover, and critically evaluate social policies like the Newfoundland Paid Family Caregiver Program, it is of equal importance that the ethics of care also supplies a useful starting place from which to imagine alternative forms of policies.

A social policy built on the foundations of the ethics of care would require a drastic reformulation of the moral subject who appears so often in social policy today. The moral subject would be a dependent person, enmeshed in relations, and oscillating through various states of vulnerability and need; the independent, self-sufficient moral subject would be abandoned. With this change, policy could not be seen as a reaction to the failing of an individual to care for herself; rather, the purpose of policy would be to ensure that the ways in which society is organized and resources are distributed enhance all caring relations.

Understanding how social organization, systems of power, and the distribution of resources effects caring relations would further involve examining structural and systemic barriers to care. Attentively assessing contexts and placing individuals within the wider social, political, economic, and cultural systems that influence our lives would 
be a necessary component of policy development and implementation. As Tronto remarks, caring requires knowledge about the lives of others $(2013,147)$, and social policies which foster the conditions necessary for care would need to be equally concerned with obtaining such knowledge. I believe that if such issues were examined, larger barriers and obstacles to care would become apparent, and policy could then be directed toward demolishing such barriers. At the same time, however, policies would need to maintain flexibility, and allow for exceptions to such patterns. That is, while there may be certain common issues that negatively affect the provision of care and caring relations in a particular context, innovative policies must maintain space "to allow for exceptions, for people whose life experiences do not fit the usual patterns" (Tronto 2013, 148), but who indeed may still be in need of caring support.

A social policy which took care as its starting point would require on-going work. It would constantly need to be scrutinized, reassessed, and evaluated, as the context in which needs arise is ever-changing and complex. It would need to be innovative and flexible. It would not be prescriptive, as the notion that one type of care fits all is nonsensical. It would also not necessarily entail the direct provision of care by the state. Instead, a social policy based on the ethics of care would involve (re)shaping the state's role so that it supports, both indirectly and directly, ongoing activities of care, which will be contextually specific and particular to our unique lives.

What I am describing above may not sound like 'policy' as we know it today. Indeed, that is the point. The ethics of care challenges us to reformulate radically the ways in which our social and economic lives are organized. It challenges us to rethink the role and the purpose of the state. It challenges us to see that we are all dependent, that 
"over the course of a lifetime, [...] those who are cared for and those who give care turn out to be the same people" (Tronto 2013, 151). It challenges us to accept that caring, the activities which maintain all our lives, must be a fundamental concern for us all. 


\section{Chapter: Conclusion}

In this thesis, I have sought to demonstrate how an assessment of the Newfoundland Paid Family Caregiver Program through a critical ethics of care lens provides many fruitful starting points from which to better understand the ways in which this program (re)structures care for the elderly in the province of Newfoundland and Labrador. This analysis highlights the fact that this program conceptualizes humans as individuals whose 'normal state' is self-sufficiency, as opposed to relational beings, who are always-already involved in and dependent on relations of care. It demonstrates that these individuals are further defined in this program by one-dimensional statuses, and that this program fails to consider the wider contexts in which caring relations reside. The ethics of care lens uncovers the ways in which the responsibility for care is being (re)distributed through this program, in what I have called the 'refamilialization' of care, as opposed to Esping-Anderson's 'defamilialization' of care. It shows that this program fails to challenge the characterization of care as a private issue. It illuminates how care is monetized under this program, and explores the consequences of the targeted structure of this program. Further, many potential pockets of tension, particularly related to how this program (re)defines the caring relation as a family-employer-employee caring relation, are identified.

Finally, and perhaps most importantly, this analysis brings into question whether or not this program effectively attends to the needs it was meant to address: while family members may provide exceptional care, there are many inconsistencies in terms of ensuring that this is so. A lack of assessment of the family caregivers and the question of whether or not training will be made available to these caregivers brings into question 
whether or not competent care will be consistently provided. An ignorance of both the particular and wider context in which the unique caring needs of the elder person arises, and narrowly defined notions of what constitutes care and respite care, indicates that the production and maintenance of a healthy caring relation that meets the specific needs of both caregivers and care recipients may not be possible. While it remains to be seen exactly how the consequences of the design of the program manifest, this normative assessment demonstrates that there are critical points that must be carefully interrogated and examined as the pilot project moves forward.

These critical points, I believe, are important sites from which to pursue future research. In terms of the Newfoundland Paid Family Caregiver Program, much work remains to be done so as to fully understand the implications and consequences of this program. Drawing upon my assessment of the Newfoundland Paid Family Caregiver Program, I wish to highlight a few directions for future research in particular.

First, I believe that work needs to be done to understand how government care policies, like the Newfoundland Paid Family Caregiver Program, formulate the moral subject. As this analysis reveals, the Newfoundland Paid Family Caregiver Program relies upon a conceptualization of a moral subject that is independent, thereby indicating that the 'normal' human state is defined as self-sufficient. This conceptualization, I believe, is both inaccurate and dangerous. As the ethics of care reminds us, we are all embedded in relations of care, and we all experience vulnerability and dependency. I think it is important to understand why the relational reality of our lives is ignored by governments and in government policy. How do governments justify such an understanding of human nature? Who does this formulation serve to benefit? Who does 
this marginalize? As an extension of this, further research is needed to deconstruct and argue against this understanding of human nature. Research needs to be undertaken that demonstrates the importance of taking caregiving and care receiving as a necessary, important, and fundamental part of our lives. Such research will challenge social policies which are formulated without serious consideration for care. As Hankivsky writes, "If we start with the assumption that the need for care should be taken seriously, then the status quo, developed in accordance with the values and priorities of a liberal justice orientation, is not acceptable" $(2004,2)$.

Secondly, I believe that research is needed to investigate the consequences of the Newfoundland Paid Family Caregiver Program. How has the refamilialization and monetization of care that occurs through this program impacted the particular, everyday lives of the caregivers and care receivers? Does this program facilitate the meeting of the caring need? Is quality care consistently provided? Do caregivers and care receivers feel that their voices are heard through this program? Are they able to be active participants in this program, or does this program 'dictate' the way in which the provision of care occurs? Primary data collection, including interviews with actual caregivers and care receivers of the program, will be required to answer such questions. However, as the provision of long-term care continues to face critical gaps in Newfoundland and Labrador, and indeed Canada at large, it is likely that more programs like the Newfoundland Paid Family Caregiver will be implemented. To ensure that such programs facilitate the establishment and maintenance of healthy caring relations, continued assessment of these programs is necessary. Further to the point, I argue that such assessments should rely on the ethics of care as a normative framework from which 
to probe such concerns; I reiterate the call to continue research in which we judge with care about care (Sevenhuijsen 2003, 182).

As a final note, in terms of my broader goal, I hope that this analysis demonstrates the usefulness of the ethics of care as a methodological tool from which to interrogate and examine social policy. The ethics of care, I believe, reflects the most fundamental of all human activities - that which is required for the continuity of life. We are all dependent and in need of care throughout all our lives, although the particular landscapes of care in which we find ourselves shift, and thus our particular caring needs shift as well. Understanding our relations of care, and how social-political-economic systems shape and are shaped by relations of care, is a fundamental task if we are to work toward the continual production, maintenance and repair of healthy and fulfilling lives. Social policy, which in the broadest sense is "a course of action or inaction chosen by public authorities to address a given problem or interrelated set of problems" (Pal 2010, 2), is highly concerned with this fundamental task. For this reason, I believe that the ethics of care should be taken seriously, and I contend that our social policies would have far greater reach and positive consequences if they were designed with consideration for the moral practices prioritized in the ethics of care. 


\section{References}

Adkin, Laurie, and Yasmeen Abu-Laban. 2008. The challenge of care: Early childhood education and care in Canada and Quebec. Studies in Political Economy 81 (Spring): 49-76.

Armstrong, Pat, and M. Patricia Connelly. 1999. Introduction: Feminism, political economy and the state; Contested terrain. In Feminism, political economy and the state: Contested terrain, ed. Pat Armstrong and M. Patricia Connelly, 1-21. Toronto: Canadian Scholars' Press Inc.

Armstrong, Pat, and Susan Braedley, eds. 2013. Troubling care. Toronto: Canadian Scholar's Press Inc.

Bailey, Alison. 1994. Mothering, diversity, and peace politics. Hypatia 9, no. 2: 188198.

------. 1995. Mothering, diversity, and peace: Comments on Sara Ruddick's feminist maternal peace politics. Journal of Social Philosophy 25, no. 1: 162-182.

Bart, Pauline. 1981. The reproduction of mothering by Nancy Chodorow review. off our backs 11, no. 1 (January): 19-23.

Belac, Bonnie. 2014. Long-term care facility nearing completion. The Telegram. March 5.

Bezanson, Kate, and Meg Luxton, eds. 2006. Social reproduction. Montreal: McGillQueen's University Press.

Botting, Ingrid. 2001. Health restructuring and privatization from women's perspective in Newfoundland and Labrador. In Exposing privatization, ed. Pat Armstrong, Carol Amaratunga, Jocelyne Bernier, Karen Grant, Ann Pederson, and Kay Willson, 4994. Aurora, ON: Garamond Press.

Bozalek, Vivienne, and Nancy R. Hooyman. 2012. Ageing and intergenerational care: Critical/political ethics of care and feminist gerontology perspectives. Agenda: Empowering Women for Gender Equity 26, no. 4: 37-47.

Brandsen, Cheryl. 2006. A public ethic of care: Implications for long-term care. In Socializing care, ed. Maurice Hamington and Dorothy C. Miller, 205-226. Lanham, MD: Rowmand and Littlefield Publishers, Inc.

Broughton, John M. 1983. Women's rationality and men's virtues: A critique of gender dualism in Gilligan's theory of moral development. Social Research 50, no. 3: 597-642. 
Card, Claudia. 1990. Caring and evil. Hypatia 5, no. 1 (Spring): 101-108.

Caregivers Out of Isolation Newfoundland. 2014. Paid family caregiver home support option announced by Minister Susan Sullivan, Health and Community Services. Caregivers Out of Isolation. http://www.caregiversoutofisolationnl.ca/?q=node/24 (accessed March 20, 2014).

CBC. 2013. Paid family caregiver program flawed, says mom. CBC News. December 12.

Chang, Kimberly A., and L.H.M. Ling. 2011. Globalization and its intimate other: Filipina domestic workers in Hong Kong. In Gender and global restructuring. Sightings, sites, and resistances. $2^{\text {nd }}$ ed. Ed. Marianne H. Marchand and Anne Sisson Runyan, 30-47. London: Routledge.

Chodorow, Nancy. 1974. Family structure and feminine personality. In Woman, culture and society, ed. Michelle Z. Rosaldo and Louise Lamphere, 43-66. Stanford: Standford University Press.

-----. 1978. The reproduction of mothering: Psychoanalysis and the sociology of gender. Berkeley: University of California Press.

-----. 1981. On 'The reproduction of mothering:' A methodological debate. Signs: Journal of Women in Culture and Society 6, no. 3 (Spring): 500-514.

Clement, Grace. 1996. Care, autonomy, and justice: Feminism and the ethic of care. Boulder, CO: Westview Press.

Collins, Patricia Hill. 1994. Shifting the center: Race, class, and feminist theorizing about motherhood. In American families: A multicultural reader, ed. Stephanie Coontz, Maya Parson, Gabrielle Raley, with Beth Vail, Tamara Anderson, and Ben Anderson, 197-217. New York: Routledge.

Connell, Raewyn. 2005. Masculinities. $2^{\text {nd }}$ ed. London: Polity Press.

------. 2010. Understanding neoliberalism. In Neoliberalism and everyday life, ed. Susan Braedley and Meg Luxton, 22-36. Montreal and Kingston: McGill-Queen's University Press.

Coser, Rose Laub. 1981. On 'The reproduction of mothering:' A methodological debate. Signs: Journal of Women in Culture and Society 6, no. 3 (Spring): 487-492.

Cott, Nancy F. 1977. The bonds of woman hood: 'Women's sphere' in New England; 1780-1835. New Haven: Yale University Press. 
Crocker, Diane. 2013. Mother sees issues with paid family caregiving. The Western Star. December 12.

Daly, Tamara. 2013. Imagining an ethos of care within policies, practices, and philosophy. In Troubling care, ed. Pat Armstrong and Susan Braedley, 33-45. Toronto: Canadian Scholars' Press Inc.

Davis, Kathy. 1992. Towards a feminist rhetoric: The Gilligan debate revisited. Women's Studies International Forum 15, no. 2: 219-231.

Day, Suzanne. 2013. The implications of conceptualizing care. In Troubling care, ed. Pat Armstrong and Susan Braedley, 21-32. Toronto: Canadian Scholars' Press Inc.

Dietz, Mary G. 1985. Citizenship with a feminist face: The problem with maternal thinking. Political Theory 13, no. 1 (February): 19-37.

DiQuinzio, Patrice. 1993. Exclusion and Essentialism in Feminist Theory: The Problem of Mothering. Hypatia 8, no. 3: 1-20.

DOHCS (Department of Health and Community Services). 2014. News release: Paid family caregiving will see increased flexibility and choice. Government of Newfoundland. March 7.

Duffy, Mignon. 2011. Making care count. London: Rutgers University Press.

Eckenwiler, Lisa A. 2012. Long-term care, globalization, and justice. Baltimore: The John Hopkins University Press.

Engster, Daniel. 2005. Rethinking care theory: The practice of caring and the obligation to care. Hypatia 20, no. 3 (Summer): 50-74.

Esping-Anderson, Gøsta. 1985. Politics against markets: The social democratic road to power. Princeton: Princeton University Press.

------. 1990. The three worlds of welfare capitalism. Cambridge: Polity Press

------ 1999. Social foundations of postindustrial economics. Oxford: Oxford University Press.

Federici, Silvia. 2012. Revolution at point zero. Oakland, CA: PM Press.

Feder Kittay, Eva. 1998. Social policy. In A companion to feminist philosophy, ed. Alison M. Jaggar and Iris Marion Young, 569. Oxford: Blackwell.

------. 2002. When caring is just and justice is caring: Justice and mental retardation. In 
The subject of care: Feminist perspectives on dependency, ed. Eva Feder Kittay and Ellen K. Feder, 257-276. London: Rowman and Littlefield Publishers, Inc.

Feder Kittay, Eva, Bruce Jennings, and Angela A. Wasunna. 2005. Dependency, difference and the global ethic of longterm care. The Journal of Political Philosophy 13, no. 4: 443-469.

Fine, Michael D. 2007. A caring society? New York: Palgrave Macmillan.

Finlay, Linda. 2002. Negotiating the swamp: The opportunity and challenge of reflexivity in research practice. Qualitative Research 2: 209-230.

Fisher, Bernice, and Joan Tronto. 1990. Toward a feminist theory of caring. In Circles of care: Work and identity in women's lives, ed. Emily K. Abel and Margaret K. Nelson, 35-62. Albany, NY: State University of New York Press.

FitzGerald Murphy, Maggie. 2014. Global care chains, commodity chains, and the valuation of care: A theoretical discussion. American International Journal of Social Science 3, no. 5 (October): 191-199.

Fox Keller, Evelyn. 1995. Reflections on gender and science. New Haven: Yale University Press.

Fraser, Nancy, and Linda Nicholson. 1989. Social criticism without philosophy: An encounter between feminism and postmodernism. Social Text 21: 83-104.

Gewertz, Deborah. 1984. The Tchambuli view of persons: A critique of individualism in the works of Mead and Chodorow. American Anthropologist 86, no. 3 (September): 615-629.

Gilligan, Carol. 1982. In a different voice. Cambridge: Harvard University Press.

-----. 1986. Reply to critics. Signs: Journal of Women in Culture and Society 11, no. 2 (Winter): 324-333.

Goodin, Robert. 1985. Protecting the vulnerable: A re-analysis of our social responsibilities. Chicago: University of Chicago Press.

-----. 1995. Utilitarianism as a public philosophy. Cambridge: Cambridge University Press.

Government of Newfoundland. 2005. Provincial home support program operational standards. St. John's, NL: Department of Health and Community Services. http://www.health.gov.nl.ca/health/publications/home_support_manual.pdf (accessed January 2, 2015). 
-----. 2012. Close to home: A strategy for long-term care and community support services 2012. St. John's, NL: Department of Health and Community Services. http://www.health.gov.nl.ca/health/long_term_care/ltc_plan.pdf (accessed February 26, 2014).

------. 2014a. Long-term care facilities listing. Department of Health and Community Services. http://www.health.gov.nl.ca/health/findhealthservices/Long Term Care Homes Contact Information.pdf (accessed March 18, 2014).

------. 2014b. Services in your region. Department of Health and Community Services. http://www.health.gov.nl.ca/health/findhealthservices/in your_community.html (accessed March 18, 2014).

-----. 2014c. Payment of family caregivers for home support services: Financial staff. St. John's, NL: Department of Health and Community Services.

-----. 2014d. Payment of family caregivers for home support services: Clinical staff. St. John's, NL: Department of Health and Community Services.

-----. 2014e. Community supports program rate. St. John's, NL: Department of Health and Community Services.

Graham, Hilary. 1993. Caring: A labour of love. In A labour of love: Women, work and caring, ed. Janet Finch and Dulcie Groves. London: Routledge.

Hall, Stuart. 1988. The toad in the garden: Thatcherism among the theorists. In Marxism and the interpretation of culture, ed. Cary Nelson and Lawrence Grossberg, 3557. London: Macmillan Education.

Hankivsky, Olena. 2004. Social policy and the ethic of care. Vancouver: UBC Press.

-----. 2006. Imagining ethical globalization: The contributions of a care ethic. Journal of Global Ethics 2, no. 1 (June): 91-110.

-----. 2011. The dark side of care: The push factors of human trafficking. In Feminist ethics and social policy: Towards a new global political economy of care, ed. Rianne Mahon and Fiona Robinson, 145-161. Vancouver: UBC Press.

------. 2014. Rethinking care ethics: On the promise and potential of intersectional analysis. American Political Science Review 108, no. 2 (May): 252-264.

Harding, Sandra. 1987. The curious coincidence of feminine and African moralities. In Women and moral theory, ed. Eva Feder Kittay and Diana T. Meyers, 296-315. Totowa, NJ: Rowman and Littlefield. 
Harrington Meyer, Madonna, ed. 2000. Care work: Gender, labour and the welfare state. New York: Routledge.

Hekman, Susan.1995. Moral voices, moral selves: Carol Gilligan and feminist moral theory. University Park, PA: The Pennsylvania State University Press.

Held, Virginia. 1987. Feminism and moral theory. In Women and moral theory, ed. Eva Feder Kittay and Diana T. Meyers, 111-129. Totowa, NJ: Rowman and Littlefield.

-----. 1989. Birth and death. Ethics 99: 362-388.

-----. 1993. Feminist morality: Transforming culture, society and politics. Chicago: University of Chicago Press.

-------, ed. 1995. Justice and care: Essential readings in feminist ethics. New York: Basic Books.

------. 2006. The ethics of care. Oxford: Oxford University Press.

Herring, Jonathan. 2014. The disability critique of care. Elder Law Review 8: 1-15.

Heyes, Cressida J. 1997. Anti-essentialism in practice: Carol Gilligan and feminist philosophy. Hypatia 12, no. 3 (Summer): 142-162.

Hoagland, Sarah Lucia. 1990. Some concerns about Nel Noddings' 'Caring'. Hypatia 5, no. 1 (Spring): 109-114.

------. 1991. Some thoughts about 'Caring'. In Feminist ethics, ed. by Claudia Card, 246263. Kansas: University Press of Kansas.

Hollway, Wendy. 2006. The capacity to care: Gender and ethical subjectivity. New York: Routledge.

hooks, bell. 2000. Feminist theory: From margin to center. $2^{\text {nd }}$ ed. Brooklyn: South End Press.

Hughes, Bill, Linda McKie, Debra Hopkins, and Nick Watson. 2005. Love's labours lost? Feminism, the Disabled People's Movement and an ethic of care. Socoiology 39, no. 2: 259-275.

Jennings, Ann L. 1993. Public or private? Institutional economics and feminism. In Beyond economic man: Feminist theory and economics, ed. Marianne A. Ferber and Julie A. Nelson, 111-129. Chicago: University of Chicago Press. 
Keller, Jean. 2010. Rethinking Ruddick and the ethnocentrism critique of Maternal Thinking. Hypatia 25, no. 4 (Fall): 834-851.

Kerber, Linda. 1986. Some cautionary words for historians. Signs: Journal of Women in Culture and Society 11, no. 2 (Winter): 304-310.

Klaver, Klaartje, and Andries Baart. 2011. Attentiveness in care: Towards a theoretical framework. Nursing Ethics 18, no. 5: 686-693.

Kohlberg, Lawrence. 1971. From is to ought: How to commit the naturalistic fallacy and get away with it in the study of moral development. In Cognitive development and psychology, ed. Theodore Mischel, 151-235. New York: Academic Press.

------. 1981. The philosophy of moral development. San Francisco: Harper and Row.

Kröger, Teppo. 2009. Care research and disability studies: Nothing in common? Critical Social Policy 29, no. 3 (August): 398-420.

Kuhn, Thomas. 1996. The structure of scientific revolutions. Chicago: The University of Chicago Press.

Kyle, Jess. 2013. Protecting the world: Military humanitarian intervention and the ethics of care. Hypatia 28, no. 2 (Spring): 257-273.

Larner, Wendy. 2000. Neo-liberalism: Policy, ideology, governmentality. Studies in Political Economy 63 (Autumn): 5-25.

Liberal Party of Newfoundland and Labrador. 2014. Paid family caregiver program finally announced two years later: Parsons. Liberal Party. http://liberaloppositio nnl. com/paid-family-caregiver-program-finally-announced-two-years-laterparsons/ (accessed March 20, 2014).

Lorber, Judith. 1981. On 'The reproduction of mothering:' A methodological debate. Signs: Journal of Women in Culture and Society 6, no. 3 (Spring): 482-486.

Luria, Zella. 1986. A methodological critique. Signs: Journal of Women in Culture and Society 11, no. 2 (Winter): 316-321.

Luxton, Meg. 1980. More than a labour of love. Toronto: The Women's Press.

------. 2006. Feminist political economy in Canada and the politics of social reproduction. In Social reproduction, ed. Kate Bezanson and Meg Luxton, 11-44. Montreal and Kingston: McGill-Queen's University Press. 
Mahon, Rianne. 2002. Gender and welfare state restructuring. In Child care policy at the crossroads, ed. Rianne Mahon and Sonya Michel, 1-30. New York: Routledge.

Mahon, Rianne, and Fiona Robinson. 2011. Introduction to Feminist ethics and social policy: Towards a new global political economy of care, ed. Rianne Mahon and Fiona Robinson, 1-17. Vancouver: UBC Press.

Marchand, Marianne H., and Anne Sisson Runyan, eds. 2011. Gender and global restructuring: Sightings, sites, and resistances. $2^{\text {nd }}$ ed. London: Routledge.

Martin, Jane Roland. 1994. Methodological essentialism, false difference, and other dangerous traps. Signs: Journal of Women in Culture and Society 19, no. 3 (Spring): 630-657.

Michael, Lorraine. 2013. Paid caregiver program presenting more problems than it solves. New Democratic Party of Newfoundland. Press release. December 10. http://nlndpcaucus.ca/news/lorraine-michael-question-period-12102013 (accessed February 24, 2014).

Milligan, Christine, and Janine Wiles. 2010. Landscapes of care. Progress in Human Geography 34, no. 6: 736-754.

Mkandawire, Thandika. 2005. Targeting and universalism in poverty reduction. Social Policy and Development Paper no. 23. Geneva: UNRISD.

Narayan, Uma. 1995. Colonialism and its others: Considerations on rights and care discourses. Hypatia 10, no. 2 (Spring): 133-140.

NDP of Newfoundland and Labrador. 2013. Paid caregiver program presenting more problems than it solves. New Democratic Party. http://nlndpcaucus.ca/nr121013 FamilyHomeCare\#sthash.mEgKUOmP.dpuf (accessed March 20, 2014).

Noddings, Nel. 1984. Caring: A feminine approach to ethics and moral education. Berkeley: University of California Press.

-----. 2012. The language of care ethics. Knowledge Quest 40, no. 5 (May-June): 52-56.

Pal, Leslie. 2010. Beyond policy analysis: Public issue management in turbulent times. $4^{\text {th }}$ ed. Toronto: Nelson Education Press.

PC Party of Newfoundland and Labrador. 2011. 2011 Policy blue book: PC Party of Newfoundland and Labrador. Progressive Conservative Party. http://newenergynl.ca/wp-content/uploads/2011/09/PC-Blue-Book_Web.pdf (accessed March 1, 2014). 
------. 2014. Paid family caregiver means choice. Progressive Conservative Party. http://newenergynl.ca/newspiece/20140307-4/ (accessed March 20, 2014).

Peterson, V. Spike. 2003. A critical rewriting of global political economy: Integrating reproductive, productive and virtual economies. London: Routledge.

Peterson, V. Spike, and Anne Sisson Runyan. 1993. Global gender issues. Boulder, CO: Westview Press.

Phillips, Judith. 2007. Care. Cambridge: Polity Press.

Rich, Adrienne. 1980. Compulsory heterosexuality and lesbian existence. Signs: Journal of Women in Culture and Society 5, no. 4 (Summer): 631-660.

Robinson, Fiona. 1999. Globalizing care: Ethics, feminist theory, and international relations. Boulder, CO: Westview Press.

------. 2006a. Beyond labour rights: The ethics of care and women's work in the global economy. International Feminist Journal of Politics 8, no. 3 (September): 321342.

-----. 2006b. Ethical globalization? States, corporations, and the ethics of care. In Socializing care, ed. Maurice Hamington and Dorothy C. Miller, 163-182. New York: Rowman and Littlefield Publishers, Inc.

------. 2011a. Care ethics and the transnationalization of care: Reflections on autonomy, hegemonic masculinities, and globalization. In Feminist ethics and social policy: Towards a new global political economy of care, ed. Rianne Mahon and Fiona Robinson, 127-144. Vancouver: UBC Press.

-----. 2011b. The ethics of care: A feminist approach to human security. Philadelphia: Temple University Press.

-----. 2013a. Discourses of motherhood and women's health: Maternal Thinking as feminist politics. Journal of International Political Theory 10, no. 1: 94-108.

------. 2013b. Global care ethics: Beyond care, beyond distribution. Journal of Global Ethics Special Issue: Critical Approaches to Global Justice; At the Frontier 9, no. 2 (May): 131-143.

Rossi, Alice S. 1981. On 'The reproduction of mothering:' A methodological debate. Signs: Journal of Women in Culture and Society 6, no. 3 (Spring): 492-500.

Ruddick, Sara. 1989. Maternal thinking: Towards a politics of peace. Boston: Beacon Press. 
Rumsey, Jean P. 1990. Constructing Maternal Thinking. Hypatia 5, no. 3 (Fall): 125-131.

Senchuk, Dennis M. 1990. Listening to a different voice: A feminist critique of Gilligan. Studies in Philosophy and Education 10, no. 3: 233-249.

Sevenhuijsen, Selma. 1998. Citizenship and the ethics of care: Feminist considerations on justice, morality, and politics. London: Routledge.

-----. 2000. Caring in the third way: The relation between obligation, responsibility and care in third way discourse. Critical Social Policy 20, no. 1: 5-37.

-----. 2003. The place of care: The relevance of the feminist ethic of care for social policy. Feminist Theory 4, no. 2: 179-197.

Sevenjuijsen, Selma, Vivienne Bozalek, Amanda Gouws, and Marie Minnaar-Mcdonald. 2006. South African social welfare policy: An analysis through the ethic of care. In Socializing care, ed. Maurice Hamington and Dorothy C. Miller, 69-87. Lanham, MD: Rowmand and Littlefield Publishers, Inc.

Sowerby, Eileen Margaret. 1993. Nel Noddings' Caring: A critical analysis. Master's thesis, University of British Columbia.

Spelman, Elizabeth V. 1988. Inessential woman: Problems of exclusion in feminist thought. Boston: Beacon Press.

Stack, Carol B. 1986. The culture of gender: Women and men of color. Signs: Journal of Women in Culture and Society 11, no. 2 (Winter): 321-324.

Sullivan, Susan. 2013. Paid family caregiving home support option will offer more choice to new home support clients. Department of Health and Community Services. Press release. December 9. http://www.releases.gov.nl.ca/ releases/2013/health/1209n02.htm (accessed February 24, 2014).

Taylor, Jill McLean, Carol Gilligan, and Amy Sullivan. 1997. Between voice and silence: Women and girls, race and relationships. Cambridge: Harvard University Press.

Tickner, J. Ann. 1997. You just don't understand: Troubled engagements between feminist and IR theorists. International Studies Quarterly 41: 611-632.

Tronto, Joan. 1987. Beyond gender difference to a theory of care. Signs: Journal of Women in Culture and Society 12, no. 4 (Summer): 644-663.

-----. 1989. Women and caring: What can feminists learn about morality from caring? In 
Gender/body/knowledge, ed. Alison M. Jagger and Susan R. Bordo, 172-187. New Brunswick, NJ: Rutgers University Press.

1993. Moral boundaries: A political argument for an ethic of care. New York: Routledge.

1995. Care as a basis for radical political judgments. Hypatia 10, no. 2: 141-149.

2011. A feminist democratic ethics of care and global care workers: Citizenship and responsibility. In Feminist ethics and social policy: Towards a new global political economy of care, ed. Rianne Mahon and Fiona Robinson, 162-177. Vancouver: UBC Press.

-----. 2013. Caring democracy: Markets, equality, and justice. New York: New York University Press.

Ungerson, Clare. 1990. The language of care: Crossing the boundaries. In Gender and caring: Work and welfare in Britain and Scandinavia, ed. Clare Ungerson, 8-33. New York: Harvester.

Urban Walker, Margaret. 1998. Moral understandings: A feminist study in ethics. New York: Routledge.

Waerness, Kari. 1996. The rationality of caring. In Caregiving: Readings in knowledge, practice, ethics, and politics, ed. Suzanne Gordon, Patricia Benner, and Nel Noddings, 231-255. Philadelphia: University of Pennsylvania Press.

Ward-Griffin, Catherine. 2008. Health professionals for aging relatives: Negotiating the public-private boundary. In Aging and caring at the intersection of work and home life: Blurring the boundaries, ed. Anne Martin-Matthews and Judith E. Philips, 1-20. New York: Lawrence Erlbaum Associates.

Williams, Fiona. 2001. In and beyond new labour: Towards a new political ethics of care. Critical Social Policy 21, no. 4: 467-493.

------. 2010. Claiming and framing in the making of care policies: The recognition and redistribution of care. Geneva: UNRISD.

-----. 2011. Towards a transnational analysis of the political economy of care. In Feminist ethics and social policy: Towards a new global political economy of care, ed. Rianne Mahon and Fiona Robinson, 21-38. Vancouver: UBC Press.

Williams, Rhonda M. 1993. Race, deconstruction, and the emergent agenda of feminist 
economic theory. In Beyond economic man: Feminist theory and economics, ed. Marianne A. Ferber and Julie A. Nelson, 144-153. Chicago: University of Chicago Press.

Wilson, Shawn. 2008. Research is ceremony. Halifax: Fernwood Publishing Press. 